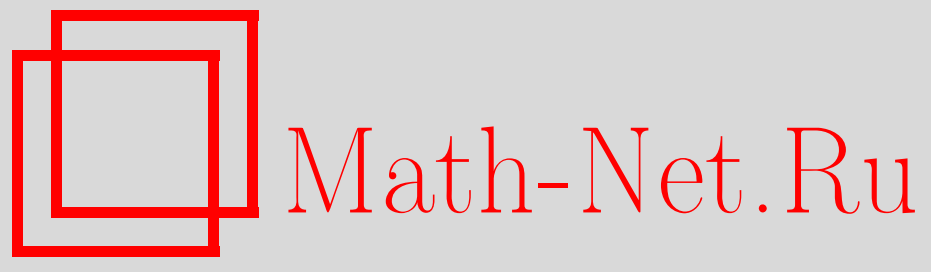

И. А. Тайманов, Сингулярные спектральные кривые в конечнозонном интегрировании, УМН, 2011, том 66, выпуск 1, 111-150

DOI: https://doi.org/10.4213/rm9403

Использование Общероссийского математического портала Math-Net.Ru подразумевает, что вы прочитали и согласны с пользовательским соглашением http://www . mathnet.ru/rus/agreement

Параметры загрузки:

IP: 3.80 .253 .173

26 апреля 2023 г., 02:29:51

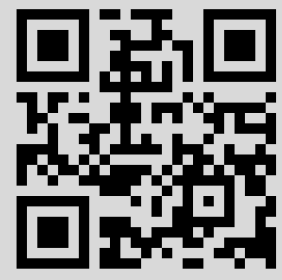




\section{Сингулярные спектральные кривые в конечнозонном интегрировании}

\section{И. А. Тайманов}

В статье на ряде примеров рассматриваются применения метода конечнозонного интегрирования нелинейных уравнений, при которых возникают спектральные кривые с особенностями. В частности, разбираются конструкции ортогональных криволинейных координат, для которых приводимая спектральная кривая состоит из рациональных компонент, и конечнозонных многообразий Фробениуса, демонстрируется солитонная деформация спектральных кривых, состоящая в возникновении и исчезновении особых точек и отвечающая уравнениям с самосогласованными источниками.

Библиография: 52 названия.

Ключевые слова: конечнозонное интегрирование, нелинейные уравнения, римановы поверхности, алгебраические кривые с особенностями.

СОДЕРЖАНИЕ

Введение .................................................... 112

1. Функции Бейкера-Ахиезера на сингулярных спектральных кривых . . 113

1.1. Спектральные кривые............................... 113

1.2. Алгебраические кривые с особенностями . . . . . . . . . . . . . . 116

2. Ортогональные криволинейные системы координат и многообразия

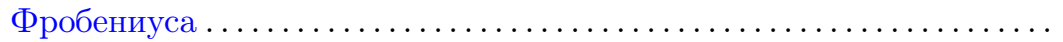

2.1. Ортогональные криволинейные координаты и интегрируемые

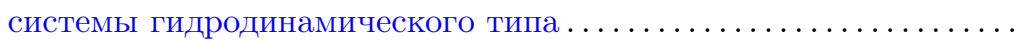

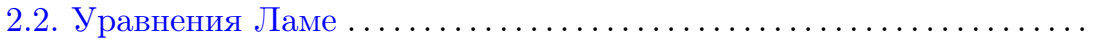

2.3. Егоровские метрики нулевой кривизны как интегрируемая система: теорема Дубровина............................

2.4. Метод Захарова построения метрик с диагональной кривизной и ортогональных криволинейных координат $\ldots \ldots \ldots \ldots \ldots \ldots$

2.5. Конструкция Кричевера конечнозонных ортогональных криво-

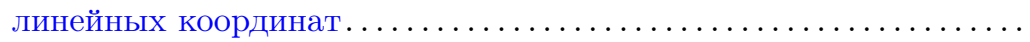

2.6. Системы координат, отвечающие сингулярным спектральным кривым ....................................... 129

2.7. Замечание о дискретных ортогональных координатах......... 134 
3. Многообразия Фробениуса ................................. 135

3.1. Уравнения ассоциативности и многообразия Фробениуса....... 135

3.2. Конечнозонные многообразия Фробениуса............... 136

4. Солитонные уравнения с самосогласованными источниками и отвечающие им деформации спектральных кривых ............... 140

4.1. Уравнения с самосогласованными источниками . . . . . . . . . . 140

4.2. Спектральные кривые погруженных торов и конформный поток 141

4.3. Конечнозонные решения уравнений КдФ и КП с самосогласованными источниками .......................... 143

5. Некоторые другие примеры интегрируемых задач с сингулярными спектральными кривыми ................................ 145

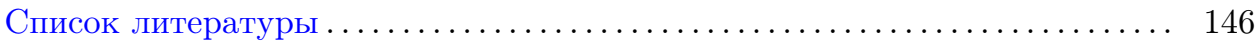

\section{Введение}

Эта статья является расширенным вариантом доклада, прочитанного автором на конференции "Geometry, Dynamics, Integrable Systems - GDIS 2010" (Сербия, 7-13 сентября 2010 г.), посвященной 60-летиям Б. А. Дубровина, В. В. Козлова, И. М. Кричевера и А. И. Нейштадта.

В докладе (и в данном тексте) мы ограничились описанием двух примеров, изученных нами совместно с А. Е. Мироновым [1], [2] и П. Г. Гриневичем [3], [4], когда в интегрируемых проблемах, возникших в дифференциальной геометрии, естественно возникают сингулярные спектральные кривые:

- при построении конечнозонных ортогональных криволинейных систем координат и решений уравнений ассочиативности [5] естественно рассмотретъ вырожденный случай, когда геометрический род спектральной кривой с особенностями равен нулю [1], [2];

в этом случае процедура построения функции Бейкера-Ахиезера и конечнозонных решений сводится к решению систем линейных уравнений, сами решения выражаются в элементарных функциях и, в частности, получаются решения уравнений ассоциативности, удовлетворяющие условиям квазиоднородности, что дает аналитическую конструкцию бесконечного класса ранее не известных многообразий Фробениуса;

- для солитонных уравнений с самосогласованными источниками, как оказалось, спектральная кривая может деформироваться, причем деформачия сводится $\kappa$ появлению и исчезновению двойных точек [3], [4].

Мы напомним основные понятия в разделе 1 , изложим эти примеры в разделах 2 и 3 соответственно и кратко укажем в разделе 4 еще несколько интересных примеров интегрируемых задач, в которых возникают сингулярные спектральные кривые.

Работа выполнена при поддержке РФФИ (грант № 09-01-12130-офи_м) и программы "Ведущие научные школы" (гран НШ-7256.2010.1). 


\section{1. Функции Бейкера-Ахиезера на сингулярных спектральных кривых}

1.1. Спектральные кривые. Определения спектральных (комплексных) кривых ${ }^{1}$ можно условно разбить на следующие три взаимно связанных типа:

1) Для одномерных дифференциальных операторов они определяются посредством блоховских функций, как это было впервые сделано в работе С. П. Новикова [6] для оператора Шрёдингера. Эта процедура включает явное построение блоховских функций.

2) Для двумерных дифференциальных операторов они определяются на заданном уровне энергии неявно, посредством “дисперсионного" соотношения между квазиимпульсами. Такие спектральные кривые были введены в работе Б. А. Дубровина, И. М. Кричевера и С. П. Новикова [7] на примере оператора Шрёдингера в магнитном поле

$$
L=\partial \bar{\partial}+A \bar{\partial}+U
$$

Для конечнозонных (на заданном уровне энергии) операторов в [7] также была решена обратная задача восстановления оператора по алгебро-геометрическим спектральным данным, включающим спектральную кривую.

3) Для задач, интегрируемых с помощью функций Бейкера-Ахиезера, введенных И. М. Кричевером [9], они определяются как римановы поверхности, на которых эти функции определены. Это включает случай, когда спектральная кривая $P(z, w)=0, P \in \mathbb{C}[z, w]$, определяется алгебраически по теореме Бурхналла-Чаунди, которая утверждает, что коммутирующие обыкновенные дифференциальные операторы $A_{1}$ и $A_{2}$ удовлетворяют алгебраическому соотношению $P\left(A_{1}, A_{2}\right)=0$.

Мы очень кратко изложим основные понятия, отсылая за подробностями к обзорам [8]-[11].

1) В конечнозонном интегрировании спектральные кривые возникли в изначальной работе Новикова [6] как римановы поверхности, параметризующие блоховские функции оператора $L=-\frac{d^{2}}{d x^{2}}+u(x)$, входящего в представление Лакса

$$
\frac{\partial L}{\partial t}=[L, A]
$$

для уравнения Кортевега-де Фриза. Блоховская бункиия оператора $L$ с периодическим потенциалом $u(x)=u(x+T)$ - это общая (формальная) собственная функция оператора $L$ и оператора $\widehat{T}$ сдвига на $T: \widehat{T}(f)(x)=f(x+T)$, т. е. решение системы уравнений

$$
L \psi=E \psi, \quad \widehat{T} \psi=e^{i p(E) T} \psi
$$

\footnotetext{
${ }^{1} \mathrm{C}$ топологической точки зрения - это римановы поверхности, но, поскольку метод конечнозонного интегрирования имеет дело с поверхностями конечного рода, в нем устоялась терминология из комплексной алгебраической геометрии.
} 
Поскольку оператор $L$ имеет порядок два, то для каждого значения $E$ он имеет двумерное пространство решений $V_{E}$ уравнения $L \psi=E \psi$, на которое ограничивается оператор $\widehat{T}$. При этом спектральная кривая Г возникает как двулистное накрытие

$$
\pi: \Gamma \rightarrow \mathbb{C}
$$

на котором блоховская функция $\psi(x, P)$ корректно определена как мероморфная функция от $P \in \Gamma$, т. е. поверхность $Г$ параметризует все блоховские функции. Поскольку $\left.\operatorname{det} \widehat{T}\right|_{V_{E}}=1$, то в точках ветвления $E$ этого накрытия собственные значения $\left.\widehat{T}\right|_{V_{E}}$ равны \pm 1 и оператор $\widehat{T}$ недиагонализируем. Если таких точек конечное число, то оператор $L$ называется конечнозонным ${ }^{2}$, а риманова поверхность $Г$ пополняется точкой ветвления на бесконечности $E=\infty$ до (комплексной) алгебраической кривой.

Напомним, что уравнение КдФ является первым в иерархии уравнений, каждое из которых представляется в виде

$$
\frac{\partial L}{\partial t_{k}}=\left[L, A_{k}\right]
$$

где $A_{k}$ - дифференциальный оператор порядка $2 k+1$, и потоки, порожденные этими уравнениями, коммутируют.

В частности, в [6] были доказаны два фундаментальных результата, лежащих в основе метода конечнозонного интегрирования:

- если потенциал и (x) является решением уравнения вида

$$
\left[L, A_{N}+c_{1} A_{N-1}+\cdots+c_{N-1} A_{1}\right]=0
$$

(уравнение Новикова), то спектральная кривая есть риманова поверхность, заданная уравнением вида $w^{2}=Q(E)$, где $Q(E)$ - многочлен степени $2 N+1$ (решение уравнения КдФ, для которых это имеет место, также называются конечнозонными);

- спектральная кривая Г и функиия квазиимпульса $p(E)$, корректно определенная на ней, с точностью до $2 \pi k / T, k \in \mathbb{Z}$, являются первыми интегралами уравнений КдФ; в частности, для конечнозонных решений первыми интегралами являются корни уравнения $Q(E)=0$, и классические интегралы Крускала-Миуры выражаются в терминах этих точек ветвления.

Для гладких вещественных решений уравнения КдФ спектральная кривая неособая и вещественная: многочлен $Q(E)$ не имеет кратных корней и все его корни вещественны.

Аналогично определяются спектральные кривые для всех одномерных (скалярных и матричных) дифференциальных и разностных операторов с периодическими коэффициентами. Построение спектральной кривой сводится к решению обыкновенных дифференциальных уравнений, к которым сводится уравнение $L \psi=E \psi($ см. (2)).

\footnotetext{
2 Эта терминология - конечнозонные операторы и блоховские функции - пришла из физики твердого тела. Позднее на английском языке термин "finite zone" был постепенно заменен на "finite gap".
} 
2) Для двумерных дифференциальных операторов $L$ с периодическими коэффициентами определим функиии Флоке как решения следующей задачи:

$$
L \psi=E \psi, \quad \psi\left(x+T_{1}, y\right)=e^{i p_{1} T_{1}} \psi(x, y), \quad \psi\left(x, y+T_{2}\right) e^{i p_{2} T_{2}} \psi(x, y),
$$

где $T_{1}$ и $T_{2}$ - периоды, а $p_{1}$ и $p_{2}-$ квазиимпульсы. Мультипликаторы функций Флоке определяются как $e^{i p_{1}}$ и $e^{i p_{2}}$.

Для гипоэллиптических операторов (например, для $\partial \bar{\partial}+\cdots$ и $\partial_{y}-\partial_{x}^{2}+\cdots$, где точками обозначены члены меньшего порядка) можно показать, что энергия $E$ и квазиимпульсы $p_{1}$ и $p_{2}$ связаны аналитическим “дисперсионным" соотношением

$$
F\left(E, p_{1}, p_{2}\right)=0
$$

Комплексная кривая в $p$-пространстве, заданная уравнением (3) при фиксированном значении $E$, называется спектральной кривой на уровне энергии $E$ [7]. Существование такой кривой (возможно, бесконечного рода) можно показать либо методами теории возмущений [12], либо с использованием теоремы Келдыша [13].

В частности, с помощью представления Вейерштрасса торов в $\mathbb{R}^{3}$ (и в $\mathbb{R}^{4}$ ) через решения уравнения $\mathscr{D} \psi=0$, где $\mathscr{D}$ - двумерный оператор Дирака с периодическими коэффициентами, в [14] была введена спектральная кривая тора, погруженного в $\mathbb{R}^{3}$, как спектральная кривая оператора $\mathscr{D}$ на нулевом уровне энергии. Как оказалось, эта спектральная кривая отражает геометрические свойства этой поверхности [13] (см. также [15]). В п. 4.2 мы обсуждаем пример, связанный со спектральными кривыми торов.

В отличие от одномерного случая спектральная кривая не может быть построена с помощью решения прямой задачи. Однако она входит в данные обратной задачи в методе функций Бейкера-Ахиезера.

3) Продемонстрируем решение обратной задачи и построение решений нелинейных уравнений с помощью функций Бейкера-Ахиезера на изначальном примере уравнения Кадомцева-Петвиашвили (КП).

Развивая технику Дубровина и Итса-Матвеева построения конечнозонных решений уравнения КдФ, И. М. Кричевер определил функиию Бейкера-Ахиезера (для уравнения КП) и с ее помощью решил обратную задачу построения решений уравнения КП по алгебро-геометрическим спектральным данным следующим образом [16].

Пусть $\Gamma$ - гладкая риманова поверхность рода $g, P$ - отмеченная точка на ней и $k^{-1}$ - локальный параметр в окрестности этой точки, $k(P)=\infty, D=\gamma_{1}+$ $\cdots+\gamma_{g}-$ эффективный дивизор степени $g$ общего положения $\left(\gamma_{1}, \ldots, \gamma_{g} \in \Gamma\right)$. Тогда

- существует и притом единственная функиия $\psi(x, y, t, \gamma), \gamma \in \Gamma$, мероморфная по $\gamma$ на $\Gamma \backslash P$, имеющая полюсы только в точках из $D$ (точнее, дивизор полюсов $\leqslant D)$ и асимптотику

$$
\psi=e^{k x+k^{2} y+k^{3} t}\left(1+\sum_{m>0} \frac{\xi_{m}(x, y, t)}{k^{m}}\right) \quad \text { nрu } \gamma \rightarrow P
$$


функция $u(x, y, t)=-2 \partial_{x} \xi_{1}$ является решением уравнения КП

$$
\frac{3}{4} u_{y y}=\frac{\partial}{\partial x}\left[u_{t}-\frac{1}{4}\left(6 u u_{x}+u_{x x x}\right)\right]
$$

при всех $\gamma \in \Gamma \backslash P$ выполняется уравнение

$$
\frac{\partial \psi}{\partial y}=L \psi, \quad \text { əде } L=\frac{\partial^{2}}{\partial x^{2}}+u,
$$

и имеет место явная формула для функции и $(x, y, t)$ в терминах тэтафункиии римановой поверхности Г:

$$
u(x, y, t)=2 \frac{\partial^{2}}{\partial x^{2}} \log \theta\left(U x+V y+W t+z_{0}\right)+c .
$$

Набор $\left(\Gamma, P, k^{-1}, D\right)$ задает “спектральные данные” Кричевера обратной задачи для решений КП или оператора $\frac{\partial}{\partial y}-L$, для которого, согласно (4), в случае периодичности потенциала $u$ функция $\psi$ является функцией Флоке на нулевом уровне энергии.

Если на Г существует мероморфная функция с единственным полюсом, который имеет второй порядок и расположен в точке $P$, то существует двулистное накрытие $\Gamma \rightarrow \mathbb{C} P^{1}$ с точкой ветвления в $P$, зависимость функции $u$ от $y$ исчезает, функция $\psi$ переходит в блоховскую функцию оператора Шрёдингера $L$, формула (5) переходит в формулу Итса-Матвеева для конечнозонных решений уравнения КдФ, а набор $\left(\Gamma, P, k^{-1}, D\right)$ - в данные обратной задачи для конечнозонного оператора Шрёдингера. Эти результаты для уравнения КдФ были получены ранее в работах Б. А. Дубровина, С. П. Новикова, А. Р. Итса, В. Б. Матвеева (см. обзор [8]).

Риманова поверхность Г называется спектральной кривой, а решения, полученные таким методом, называются конечнозонными. При этом не исключаются и квазипериодические решения (как легко заметить из (5), они могут получаться таким методом). Эта терминология применяется ко всем задачам, решаемым с помощью функций Бейкера-Ахиезера (методом конечнозонного интегрирования).

Вещественные периодические гладкие решения уравнений КдФ, КП и sineGordon строятся по гладким римановым поверхностям. Тэта-функциональные формулы устроены достаточно сложно, поскольку входящие в них параметры связаны трансцендентными уравнениями.

В то же время для некоторых других уравнений, подпадающих под метод конечнозонного интегрирования, интересные решения строятся по сингулярным спектральным кривым, в том числе с геометрическим родом равным нулю. В последнем случае решение выражается через элементарные функции и допускает простое качественное исследование (см. разделы 2 и 3).

1.2. Алгебраические кривые с особенностями. Напомним некоторые основные понятия, касающиеся комплексных алгебраических кривых с особенностями (сингулярных кривых); подробности см. в [17; гл. 4]). 
Пусть Г - комплексная алгебраическая кривая с особенностями.

Тогда существует такой морфизм гладкой алгебраической кривой $\Gamma_{\mathrm{nm}}$ :

$$
\pi: \Gamma_{\mathrm{nm}} \rightarrow \Gamma
$$

что

1) в $\Gamma_{\mathrm{nm}}$ выделено конечное множество точек $S$, разбитое на подклассы, и отображение $\pi$ переводит $S$ в точности в множество особых точек Sing = Sing $\Gamma$ кривой $\Gamma$, причем прообраз каждой точки из Sing состоит из некоторого подкласса точек из $S$;

2) отображение $\pi: \Gamma_{\mathrm{nm}} \backslash S \rightarrow \Gamma \backslash$ Sing является гладкой взаимно однозначной проекцией;

3) любое регулярное отображение $F: X \rightarrow \Gamma$ несингулярного алгебраического многообразия $X$ с всюду плотным образом $F(X) \subset \Gamma$ пропускается через $\Gamma_{\mathrm{nm}}$, т. е. $F=\pi G$ для некоторого регулярного отображения $G: X \rightarrow \Gamma_{\mathrm{nm}}$.

Отображение $\pi$ с такими свойствами называется нормализацией кривой $\Gamma$ и определяется этими условиями однозначно. Иногда нормализацией называют саму кривую $\Gamma_{\text {nm }}$.

Род кривой $\Gamma_{\mathrm{nm}}$ называется геометрическим родом кривой $Г$ и обозначается через $p_{g}(\Gamma)$.

В формулу Римана-Роха входит арифметический род $p_{a}(\Gamma)$, который складывается из геометрического рода и положительного вклада сингулярностей (точек из Sing). Для гладкой кривой мы имеем $p_{a}=p_{g}$.

Мероморфная 1-форма $\omega$ на $\Gamma_{\mathrm{nm}}$ задает регулярный дифференииал на $\Gamma$, если для любой точки $P \in \operatorname{Sing}$ мы имеем

$$
\sum_{\pi^{-1}(P)} \operatorname{Res}(f \omega)=0
$$

для всех мероморфных функций $f$ на $\Gamma_{\mathrm{nm}}$, которые опускаются до функций на $\Gamma$, т. е. принимают в точках из каждого дивизора $D_{i}$ одно и то же значение и не имеют полюсов в $\pi^{-1}(P)$. Регулярные дифференциалы могут иметь полюсы в прообразах особых точек. Размерность пространства регулярных дифференциалов равна $p_{a}(\Gamma)$.

Пусть на неприводимой алгебраической кривой $\Gamma_{\mathrm{nm}}$ выделены $s$ наборов $D_{1}, \ldots, D_{s}$, состоящих из $r_{1}, \ldots, r_{s}$ точек, все из которых различны, и $\Gamma$ получается склейкой точек из каждого набора в одну. Тогда

$$
p_{a}(\Gamma)=p_{g}(\Gamma)+\sum_{i=1}^{s}\left(r_{i}-1\right) .
$$

Сформулируем теорему Римана-Роха для алгебраических кривых с особенностями:

- Пусть $L(D)$ - пространство мероморфных функиий на Г с полюсами в точках из $D=\sum n_{P} P$ порядка $\leqslant n_{P} u \Omega(D)$ - пространство регулярных дифференциалов на $\Gamma$, имеющих в каждой точке $P \in$ Sing нуль порядка не менъше, чем $n_{P}$. Тогда

$$
\operatorname{dim} L(D)-\operatorname{dim} \Omega(D)=\operatorname{deg} D+1-p_{a}(\Gamma) .
$$


Для дивизора общего положения $D$, где $\operatorname{deg} D \geqslant p_{a}$, мы имеем

$$
\operatorname{dim} \Omega(D)=0 \quad u \quad \operatorname{dim} L(D)=\operatorname{deg} D+1-p_{a}(\Gamma) .
$$

Стандартная схема доказательства единственности функции Бейкера-Ахиезера основана на теореме Римана-Роха, и род $g$ гладкой спектральной кривой $Г$ входит во все рассуждения как арифметический род. При работе с сингулярными спектральными кривыми достаточно во всех аргументах и определениях заменить $g$ на $p_{a}$.

Приведем простейшие примеры одномерных конечнозонных операторов Шрёдингера с сингулярными спектральными кривыми.

ПримеР 1 (кривая с двойной точкой). Пусть $\Gamma_{\mathrm{nm}}=\mathbb{C} P^{1}=\mathbb{C} \cup\{\infty\}$ с параметром $k$. Построим кривую $\Gamma$, склеив на $\Gamma_{\text {nm }}$ точки $k= \pm \lambda$. Мы имеем $p_{a}(\Gamma)=1$ и поэтому положим $D=\{k=0\}$. По спектральным данным $(\Gamma, \infty, k, D)$ строится оператор Шрёдингера ${ }^{3}$

$$
L=-\frac{d^{2}}{d x^{2}}+\frac{2 \lambda^{2}}{\sin ^{2}(\lambda x)}
$$

и функция Бейкера-Ахиезера

$$
\psi(k, x)=\left(1+\frac{i \lambda}{k} \frac{\cos (k x)}{\sin (k x)}\right) e^{i k x} .
$$

ПримеР 2 (каспидальная кривая). Пусть Г - та же, что и в предыдущем примере. Кривая Г, гомеоморфная $\Gamma_{\mathrm{nm}}$, но с каспидальной особенностью в точке $k=0$, получается, если мы ограничим класс локально голоморфных функций на $\Gamma_{\mathrm{nm}}$, считая, что голоморфная в окрестности $k=0$ функция $f$ на $\Gamma_{\mathrm{nm}}$ голоморфна в окрестности точки $k=0$ на Г тогда и только тогда, когда

$$
\left.f^{\prime}\right|_{k=0}=0 \text {. }
$$

Мы имеем $p_{a}(\Gamma)=1$. Спектральные данные $(\Gamma, \infty, k, D=\{k=0\})$ задают потенциал

функция

$$
u(x)=\frac{2}{x^{2}}
$$

$$
\psi_{1}(k, x)=\left(-\frac{\partial}{\partial x}+\frac{1}{x}\right) e^{i k x}=\left(-i k+\frac{1}{x}\right) e^{i k x}
$$

параметризует все блоховские функции и удовлетворяет условию (6). После деления ее на $-i k$ мы получаем функцию Бейкера-Ахиезера, нормированную асимптотикой при $k \rightarrow \infty$ :

$$
\psi(k, x)=\left(1+\frac{i}{k x}\right) e^{i k x} .
$$

Этот потенциал получается из потенциалов, указанных в примере 1 , в пределе $\lambda \rightarrow 0$.

\footnotetext{
${ }^{3}$ Его запись отличается от (4) знаком при $d^{2} / d x^{2}$, что отражено в изменении асимптотики функции $\psi: \psi \approx e^{i k x}$ при $k \rightarrow \infty$.
} 
ПримеР 3. Потенциал из примера 2 входит в серию рациональных солитонных потенциалов

$$
u_{l}(x)=\frac{l(l+1)}{x^{2}} .
$$

Функция Бейкера-Ахиезера для оператора с потенциалом (7) определена на кривой $\Gamma$, которая получается из $\Gamma_{\mathrm{nm}}=\mathbb{C} P^{1}$ наложением условия, аналогичного условию (6):

$$
f^{\prime}=f^{\prime \prime}=\cdots=f^{(l)}=0 \quad \text { при } k=0 .
$$

Мы имеем $p_{a}(\Gamma)=l, D=l Q$, где $Q=\{k=0\}$, а другие спектральные данные те же, что и в примере 2. Функция Бейкера-Ахиезера имеет вид

$$
\psi_{l}(k, x)=\frac{1}{(-i k)^{l}}\left(-\frac{\partial}{\partial x}+\frac{l}{x}\right) \cdots\left(-\frac{\partial}{\partial x}+\frac{1}{x}\right) e^{i k x} .
$$

Риманова поверхность Г задается уравнением $y^{2}=x^{2 l+1}$, и нормализация имеет вид

$$
\mathbb{C} \rightarrow \Gamma \backslash\{\infty\}, \quad t \rightarrow\left(x=t^{2}, y=t^{2 n+1}\right) .
$$

Обычно функция Бейкера-Ахиезера строится как функция $\psi$ на нормализации $\Gamma_{n m}$ кривой, а кратные точки и каспидальные особенности задаются дополнительными условиями вида

$$
\psi\left(Q_{1}\right)=\psi\left(Q_{2}\right), \quad \psi^{\prime}(Q)=\cdots=\psi^{(l)}(Q) .
$$

В то же время в теории солитонов рассматривались более общие ограничения вида

$$
\left.\sum_{j=1}^{M} \sum_{i=1}^{m_{j}} a_{i j} \psi^{(j)}(k, x, \ldots)\right|_{k=Q_{j}}=0, \quad Q_{1}, \ldots, Q_{M} \in \Gamma_{\mathrm{nm}},
$$

или даже системы таких соотношений (см., например, [18]). В этом случае $\psi$ является сечением некоторого пучка над кривой $Г$, которую можно найти следующим образом. Очевидно, что в общем положении условие вида (8), наложенное на рациональные функции на $\Gamma_{\mathrm{nm}}$, не выделяет никакого подполя: для произведения двух функций, ему удовлетворяющих, соотношение не обязано выполняться. В то же время сечения искомого пучка образуют модуль над полем рациональных функций на $Г$. Пусть $f$ - такая функция и $\psi$ - сечение пучка, заданного формулой (8), тогда минимальные требования, которым должна удовлетворять функция $f$, чтобы $f \psi$ удовлетворяло (8) для всех сечений, состоят в следующем:

$$
f\left(Q_{1}\right)=\cdots=f\left(Q_{M}\right), \quad f^{\prime}\left(Q_{j}\right)=\cdots=f^{\left(m_{j}\right)}\left(Q_{j}\right)=0, \quad j=1, \ldots, M,
$$

т. е. Г получается из $\Gamma_{\mathrm{nm}}$ склейкой точек $Q_{1}, \ldots, Q_{M}$, при этом каждая из этих точек с $m_{j} \geqslant 1$ является сингулярной (см. пример 3 ).

Однако некоторые такие функции Бейкера-Ахиезера (по-видимому, все) могут быть получены вырождением из функций на гладких спектральных кривых. Приведем следующий пример. 
ПримеР 4 (А.Е. Миронов). Пусть $\Gamma_{1}-$ связная алгебраическая кривая рода $g$, на которой строится функция Бейкера-Ахиезера $\psi$. Предположим, для простоты, что $\psi$ строится по спектральным данным для уравнения КП, но удовлетворяет дополнительному условию

$$
\psi\left(Q_{1}\right)=\alpha \psi\left(Q_{2}\right)
$$

где $\alpha \neq 1$. Из теоремы Римана-Роха следует, что условие на дивизор полюсов $\psi$ - он состоит из $g+1$ точки $\gamma_{1}, \ldots, \gamma_{g+1}$ общего положения с учетом кратности - вместе с асимптотикой в существенно особой точке задает ее однозначно. Рассмотрим рациональную кривую $\Gamma_{2}=\mathbb{C} P^{1}$, на которой выделены точки $a$ и $b$, отличные от $\infty$; и отождествив точку $Q_{1}$ с $a$, а $Q_{2}-$ с $b$, получим приводимую кривую $\Gamma=\Gamma_{1} \cup \Gamma_{2} /\left\{Q_{1} \sim a, Q_{2} \sim b\right\}$ с арифметическим родом $p_{a}(\Gamma)=g+1$. Построим на $\Gamma$ функцию Бейкера-Ахиезера $\widetilde{\psi}$ с $g+2$ полюсами в точках $\gamma_{1}, \ldots, \gamma_{g+1} \in \Gamma_{1}$ и такой точке $q \in \Gamma_{2}$, что $(b-q) /(a-q)=\alpha$. Число полюсов на единицу больше арифметического рода, и мы наложим дополнительную нормировку $\psi(\infty)=0$, где $\infty \in \Gamma_{2}$. Тогда ограничение $\psi$ на компоненту $\Gamma_{2}$ будет функцией $\varphi=c /(z-q), c=$ const $\neq 0$. Согласно выбору $q$ мы имеем $\varphi(a)=\alpha \varphi(b)$, и, следовательно, ограничение $\widetilde{\psi}$ на $\Gamma_{1}$ будет искомой функцией $\psi$. Риманова поверхность $Г$ получается вырождением гладких поверхностей при стягивании двух контуров в точки $Q_{1} \sim a$ и $Q_{2} \sim b$, а функция $\widetilde{\psi}-$ вырождением функций Бейкера-Ахиезера на них. Поскольку обратная задача решается по асимптотикам $\psi$ в точках с существенными особенностями, а таких на компоненте $\Gamma_{2}$ нет, то ограничение $\widetilde{\psi}$ на компоненту $\Gamma_{2}$ несущественно. В то же время настоящей спектральной кривой, на которой $\widetilde{\psi}$ получается как функция Бейкера-Ахиезера, является приводимая алгебраическая кривая Г, a не $\Gamma_{1}$.

По-видимому, так получаются потенциальные операторы Шрёдингера из $[19]^{4}$, которые строятся по сингулярным спектральным кривым с нечетным арифметическим родом (для гладких спектральных кривых род в этой задаче всегда четен [12]). В [20] Т. М. Маланюк построил конечнозонные решения уравнения КПII, для которых спектральная кривая приводима и существенная особенность функции Бейкера-Ахиезера лежит на рациональной компоненте. Эти решения являются нелинейными суперпозициями солитонных валов.

Легко заметить, что конструкция из примера 4 обобщается на любые соотношения вида $\alpha_{1} \psi\left(Q_{1}\right)+\cdots+\alpha_{M} \psi\left(Q_{M}\right)=0$ с коэффициентами $\alpha_{1}, \ldots, \alpha_{M}$ общего положения. При этом $\Gamma$ получается из $\Gamma_{1}$ добавлением рациональной кривой $\mathbb{C} P^{1}$, пересекающейся с $\Gamma_{1}$ по точкам $Q_{1}, \ldots, Q_{M}$.

\section{2. Ортогональные криволинейные системы координат и многообразия Фробениуса}

\section{1. Ортогональные криволинейные координаты и интегрируемые} системы гидродинамического типа. В теории интегрируемых систем ортогональные криволинейные системы координат появились с двух сторон: они

\footnotetext{
4Эти примеры были построены в начале 1990-х годов, и тогда же подобные аргументы о получении их спектральных кривых при вырождении высказывал Т. М. Маланюк.
} 
естественно возникли в задаче об интегрируемости одномерных систем гидродинамического типа Дубровина-Новикова [21]-[23] и они же возникли в результате дифференциальной редукции, как частный случай метрик с диагональной кривизной, к явному построению которых метод обратной задачи был применен Захаровым [24].

Ортогональной криволинейной системой координат в римановом многообразии называется система координат $\left(u^{1}, \ldots, u^{N}\right)$, в которой метрический тензор принимает диагональный вид:

$$
d s^{2}=\sum_{i=1}^{N} H_{i}^{2}(u)\left(d u^{i}\right)^{2} .
$$

Отметим, что при $N \geqslant 4$ не каждая риманова метрика локально допускает ортогональные криволинейные координаты. В действительности, существование таких координат является очень сильным условием на метрику.

Коэффициенты $H_{i}$ называются коэффициентами Ламе, а выражения

$$
\beta_{i j}=\frac{1}{H_{j}} \frac{\partial H_{i}}{\partial u^{j}}
$$

задают коэффиииенты вращения.

Метрика называется егоровской, если локально она допускает ортогональные криволинейные координаты с симметрическими коэффициентами вращения:

$$
\beta_{i j}=\beta_{j i}, \quad 1 \leqslant i, j \leqslant N \text {. }
$$

В этом случае метрика является потенциальной:

$$
g_{i i}=H_{i}^{2}=\frac{\partial V}{\partial u^{i}}, \quad i=1, \ldots, N,
$$

для некоторого потенциала $V(u)$.

В теории систем гидродинамического типа случай римановых метрик ничем не выделен и рассматриваются общие псевдоримановы метрики, для которых метрический тензор в ортогональных координатах принимает вид

$$
d s^{2}=\sum_{i=1}^{n} \varepsilon_{i} H_{i}^{2}(u)\left(d u^{i}\right)^{2}, \quad \varepsilon_{i}= \pm 1,
$$

коэффициенты вращения определяются так же, как и для римановых метрик, а условие того, что метрика является егоровской, записывается как

$$
\beta_{i j}=\varepsilon_{i} \varepsilon_{j} \beta_{j i}
$$

Одномерная система гидродинамического типа - это эволюционная система вида

$$
u_{t}^{i}=\sum_{j=1}^{N} v_{j}^{i}(u) u_{x}^{j}, \quad i=1, \ldots, N
$$


где $u^{1}(x, t), \ldots, u^{N}(x, t)$ - функции от одномерной переменной $x$ и времени $t$. Такая система гамильтонова, если она допускает представление в виде

$$
u_{t}^{i}=\left\{u^{i}(x), \widehat{H}\right\}
$$

где

$$
\widehat{H}(u)=\int H(u) d x
$$

и скобки Пуассона (гидродинамического типа, или, как сейчас говорят, скобки Пуассона-Дубровина-Новикова) имеют вид

$$
\left\{u^{i}(x), u^{j}(y)\right\}=g^{i j}(u(x)) \delta^{\prime}(x-y)-g^{i s} \Gamma_{s k}^{j} u_{x}^{k} \delta(x-y), \quad g^{i j}=g^{j i} .
$$

Эти понятия были введены Б.А. Дубровиным и С.П. Новиковым [20], [22], доказавшими, что

1) выражение (11) с невырожденной псевдоримановой метрикой $g^{i j}$ (с верхними индексами) в $N$-мерном $u$-пространстве задает скобки Пуассона тогда и только тогда, когда эта метрика - плоская (имеет нулевую кривизну) и $\Gamma_{j k}^{i}-$ отвечающая ей связность Леви-Чивиты;

2) гамильтоновы системы гидродинамического типа возникают в результате усреднения таких интегрируемых систем, как уравнения КдФ и sine-Gordon, нелинейного уравнения Шрёдингера (НШ).

Новиковым была высказана гипотеза, что гамильтоновы системы гидродинамического типа с диагональной матрицей $\left(v_{j}^{i}\right)$ интегрируемы. Для таких систем координаты $u^{1}, \ldots, u^{N}$ называются инвариантами Римана, и матрица $\left(g^{i j}\right)$ тоже является диагональной в $u$-пространстве с ортогональными криволинейными координатами $u^{1}, \ldots, u^{N}$. Эта гипотеза была доказана С. П. Царевым, предложившим и процедуру интегрирования - обобщенный метод годографа [25].

Интерес к егоровским метрикам в рамках теории интегрируемых систем возник благодаря Дубровину, показавшему, что если плоская метрика $g^{i j}$, записанная в инвариантах Римана, является егоровской, то соответствующая гамильтонова система гидродинамического типа обладает повышенной интегрируемостью $[26]^{5}$.

В [28] предложено следующее нелокальное обобщение скобок Пуассона-Дубровина-Новикова:

$$
\left\{u^{i}(x), u^{j}(y)\right\}=g^{i j}(u(x)) \delta^{\prime}(x-y)-g^{i s} \Gamma_{s k}^{j} u_{x}^{k} \delta(x-y)+\frac{c}{2} \operatorname{sgn}(x-y) u_{x}^{i} u_{y}^{j},
$$

где $c=$ const, и было доказано, что это выражение задает скобки Пуассона тогда и только тогда, когда $\Gamma_{j k}^{i}-$ связность Леви-Чивиты, отвечающая метрике $g^{i j}$ постоянной секционной кривизны, равной $c$, и что обобщенный метод годографа также применим к гамильтоновым системам с такой скобкой Пуассона. При этом инварианты Римана задают ортогональные криволинейные координаты в пространстве постоянной кривизны $c$.

\footnotetext{
53а современным состоянием таких систем мы отсылаем читателя к [27] и библиографии в этой работе.
} 
2.2. Уравнения Ламе. В 19 -м веке теория ортогональных криволинейных систем координат была активным объектом исследований ведущих геометров (Дюпен, Гаусс, Ламе, Бианки, Дарбу). Задача классификации таких координат была в основном решена к началу 20-го века и состояние теории на этот момент времени было подытожено в монографии Дарбу [29].

Все такие системы координат строятся по решениям уравнений Ламе следующим образом.

Разобьем нетривиальные компоненты тензора кривизны Римана $R_{i j k l}$ на три группы:

1) все индексы $i, j, k, l$ попарно различны;

2) $R_{i j i k}$, где индексы $i, j, k$ попарно различны;

3) $R_{i j i j}$ с $i \neq j$.

Напомним, что тензор Римана кососимметричен по первой и второй парам индексов: $R_{i j k l}=-R_{j i k l}=-R_{i j l k}$ и $R_{i j k l}=R_{k l i j}$ для всех $i, j, k, l$.

Для диагонализируемых метрик (9) все компоненты первого типа равны нулю, равенство нулю компонент второго типа эквивалентно системе

$$
\frac{\partial^{2} H_{i}}{\partial u^{j} \partial u^{k}}=\frac{1}{H_{j}} \frac{\partial H_{j}}{\partial u^{k}} \frac{\partial H_{i}}{\partial u^{j}}+\frac{1}{H_{k}} \frac{\partial H_{k}}{\partial u^{j}} \frac{\partial H_{i}}{\partial u^{k}}, \quad i \neq j \neq k,
$$

а уравнения

$$
\frac{\partial}{\partial u^{j}}\left(\frac{1}{H_{j}} \frac{\partial H_{i}}{\partial u^{j}}\right)+\frac{\partial}{\partial u^{i}}\left(\frac{1}{H_{i}} \frac{\partial H_{j}}{\partial u^{i}}\right)+\sum_{k \neq i \neq j} \frac{1}{H_{k}^{2}} \frac{\partial H_{i}}{\partial u^{k}} \frac{\partial H_{j}}{\partial u^{k}}=0, \quad i \neq j,
$$

эквивалентны равенству нулю компонент третьего типа: $R_{i j i j}=0$.

Системы (12) и (13) состоят из $N(N-1)(N-2) / 2$ и $N(N-1) / 2$ уравнений соответственно. Из подсчета числа уравнений и неизвестных видно, что система уравнений (12), (13) на коэффициенты Ламе сильно переопределена.

Порядок системы (12), (13) можно понизить, перейдя к коэффициентам вращения. В этом случае уравнения (12) примут вид

$$
\frac{\partial \beta_{i j}}{\partial u^{k}}=\beta_{i k} \beta_{k j}, \quad i \neq j \neq k,
$$

а уравнения (13) переписываются как система

$$
\frac{\partial \beta_{i j}}{\partial u^{i}}+\frac{\partial \beta_{j i}}{\partial u^{j}}+\sum_{k \neq i, j} \beta_{k i} \beta_{k j}=0, \quad i \neq j .
$$

Уравнения (14) и (15) образуют систему уравнений Ламе и уравнения (14) есть не что иное, как условие совместности уравнений (10). Общее решение этих уравнений зависит от $N(N-1) / 2$ произвольных функций от двух переменных.

По заданному решению $\beta_{i j}$ уравнений Ламе коэффициенты Ламе находятся из уравнений (10) как решение задачи Коши

$$
H_{i}\left(0, \ldots, 0, u^{i}, 0, \ldots, 0\right)=h_{i}\left(u^{i}\right) .
$$


При этом решение зависит от начальных данных $-N$ функций $h_{i}$ одной переменной.

Задание евклидовых координат $x^{1}, \ldots, x^{N}$ как функций от $u^{1}, \ldots, u^{N}$ (проблема погружения) сводится к решению переопределенной системы линейных уравнений

$$
\frac{\partial^{2} x^{k}}{\partial u^{i} \partial u^{j}}=\sum_{l=1}^{N} \Gamma_{i j}^{l} \frac{\partial x^{k}}{\partial u^{l}},
$$

где символы Кристоффеля имеют вид

$$
\Gamma_{i j}^{k}=0, \quad i \neq j \neq k ; \quad \Gamma_{k j}^{k}=\frac{1}{H_{k}} \frac{\partial H_{k}}{\partial u^{j}} ; \quad \Gamma_{i i}^{k}=-\frac{H_{i}}{\left(H_{k}\right)^{2}} \frac{\partial H_{i}}{\partial u^{k}}, \quad i \neq k .
$$

Система уравнений (16) совместна в силу (12) и (13) и определяет ортогональную криволинейную систему координат с точностью до движений $\mathbb{R}^{N}$.

\section{3. Егоровские метрики нулевой кривизны как интегрируемая си-} стема: теорема Дубровина. В [26] Б. А. Дубровин показал, что задача построения плоских егоровских метрик интегрируется методами теории солитонов, а именно имеет место следующая теорема.

Теорема 1 [26]. 1) Егоровская (риманова или псевдориманова) метрика является плоской тогда и только тогда, когда ее коэфбициенты вращения удовлетворяют уравнениям

$$
\begin{gathered}
\frac{\partial \beta_{i j}}{\partial u^{k}}=\beta_{i k} \beta_{k j}, \quad \text { где } i, j, k \text { различны }, \\
\sum_{k=1}^{N} \frac{\partial \beta_{i j}}{\partial u^{k}}=0, \quad i \neq j .
\end{gathered}
$$

2) Система, состоящая из уравнений (17) и (18), представляется в виде условия совместности систем линейных уравнений

$$
\begin{gathered}
\frac{\partial \psi_{i}}{\partial u^{j}}=\beta_{i j} \psi_{j}, \quad i \neq j, \\
\sum_{k=1}^{N} \frac{\partial \beta_{i j}}{\partial u^{i}}=\lambda \psi_{i}, \quad i=1, \ldots, N,
\end{gathered}
$$

где $\lambda$ - спектральный параметр.

3) Для плоской егоровской метрики ограничение коэфбищиентов вращения $\beta_{i j}(u)$ на любую плоскость $u^{i}=a^{i} x+c^{i} t$ удовлетворяет системе уравнений $N$ волн:

$$
\left[A, B_{t}\right]-\left[C, B_{x}\right]=[[A, B],[C, B]]
$$

¿əe

$$
A=\operatorname{diag}\left(a^{1}, \ldots, a^{N}\right), \quad C=\operatorname{diag}\left(c^{1}, \ldots, c^{N}\right), \quad B=\left(\beta_{i j}\right),
$$

с дополнительной редукиией

$$
\operatorname{Im} B=0, \quad B^{\top}=J B J, \quad J=\operatorname{diag}\left(\varepsilon_{1}, \ldots, \varepsilon_{N}\right)
$$

(для римановых метрик все $\varepsilon_{i}$ равны 1). 
Эта теорема доказывается прямой вычислительной проверкой ее утверждений.

\section{4. Метод Захарова построения метрик с диагональной кривизной} и ортогональных криволинейных координат. В [24] В. Е. Захаров применил метод обратной задачи для построения широкого класса метрик с диагональной кривизной, к изложению которого мы перейдем.

Тензор кривизны Римана можно интерпретировать как оператор кривизны на пространстве касательных бивекторов. Пусть $M^{N}$ - риманово (или псевдориманово) многообразие, $\Lambda^{2} T M^{N}$ - векторное расслоение над $M^{N}$, слоем которого над любой точкой является пространство бивекторов в этой точке. Метрика на $M^{N}$ задает естественную метрику в слоях: если $e_{1}, \ldots, e_{N}$ - ортонормированный базис в $T_{x} M^{N}$, касательном пространстве в точке $x$, то $\left\{e_{i} \wedge e_{j}, i<j\right\}-$ ортонормированный базис в $\Lambda^{2} T_{x} M^{N}$. Тогда тензор кривизны Римана $R_{i j k l}$ задает оператор кривизны $R$ по формуле

$$
\langle R \xi, \eta\rangle=R_{A B} \xi^{A} \eta^{B}, \quad R_{A B}=R_{i j k l}, \quad A=[i j], \quad B=[k l],
$$

где $\xi=\sum_{A} e_{A} \xi^{A}, \eta=\sum_{B} e_{B} \eta^{B}-$ разложение бивекторов по базису $e_{A}=e_{i} \wedge e_{j}$, $i<j$. Заметим, что известная в теории гравитации классификация Петрова типов четырехмерных решений уравнения Эйнштейна основана на классификации алгебраических типов оператора кривизны ${ }^{6}$.

В [24] пространства с диагональной кривизной определены как пространства, у которых в окрестности каждой точки метрика диагонализируется приводится к виду $(9)$ - и в данных координатах квадратичная форма $R_{A B}$ (или оператор кривизны $R$ ) тоже диагональны.

Уравнения (17) в точности описывают коэффициенты вращения метрик с диагональной кривизной и записываются в виде

$$
\sum_{i, j, k} \varepsilon_{i j k}\left(I_{j} \frac{\partial B}{\partial u^{i}} I_{k}-I_{i} B I_{j} B I_{k}\right)=0
$$

где $B(u)=\left(\beta_{i j}\right)$ есть $(N \times N)$-матричная функция, составленная из коэффициентов вращения, а $I_{j}=\operatorname{diag}(0, \ldots, 0,1,0, \ldots, 0)$ (единица на $j$-м месте).

Рассмотрим вспомогательную функцию $\widetilde{B}=\widetilde{B}(u, s)$ (где $s=u^{N+1}$ - дополнительная переменная), удовлетворяющую уравнениям

$$
\frac{\partial}{\partial u^{j}}\left[I_{i}, \widetilde{B}\right]-\frac{\partial}{\partial u^{i}}\left[I_{j}, \widetilde{B}\right]+I_{i} \frac{\partial \widetilde{B}}{\partial s} I_{j}-I_{j} \frac{\partial \widetilde{B}}{\partial s} I_{i}-\left[\left[I_{i}, \widetilde{B}\right],\left[I_{j}, \widetilde{B}\right]\right]=0,
$$

где $i, j=1, \ldots, N+1$ и $I_{N+1}$ - единичная матрица. Если $\widetilde{B}$ удовлетворяет $(20)$, то при любом заданном значении $s$ матричная функция $\widetilde{B}$ является решением задачи $N$ волн (19).

\footnotetext{
${ }^{6}$ Сейчас, однако, под классификацией Петрова часто понимают аналогичную классификацию, основанную на алгебраических типах оператора Вейля, который таким же образом строится по тензору (конформной кривизны) Вейля.
} 
Система (20) допускает представление Лакса:

$$
\left[L_{i}, L_{j}\right]=0, \quad L_{j}=\frac{\partial}{\partial u^{j}}+I_{j} \frac{\partial}{\partial s}+\left[I_{j}, \widetilde{B}\right] .
$$

Применим к ней метод одевания, а именно, рассмотрим интегральное уравнение типа Марченко:

$$
K\left(s, s^{\prime}, u\right)=F\left(s, s^{\prime}, u\right)+\int_{s}^{\infty} K(s, q, u) F\left(q, s^{\prime}, u\right) d q
$$

где $F\left(s, s^{\prime}, u\right)$ - матричная функция.

Теорема 2 [24]. Пусть $F\left(s, s^{\prime}, u\right)$ удовлетворяет следующим условиям:

а) выполняется уравнение

$$
\frac{\partial F}{\partial u^{i}}+I_{i} \frac{\partial F}{\partial s} F+\frac{\partial F}{\partial s^{\prime}} F I_{i}=0
$$

б) уравнение (21) имеет единственное решение. Тогда функиия

$$
\widetilde{B}(s, u)=K(s, s, u)
$$

удовлетворяет уравнениям (20) и, следовательно, при любом фиксированном значении s функция $B(u)=\widetilde{B}(s, u)$ удовлетворяет (19) и задает коэффициентьи вращения метрики с диагональной кривизной.

Класс метрик с диагональной кривизной достаточно широк и, например, как отметил Захаров, к ним относятся многие известные решения уравнений Эйнштейна (в том числе метрика Шварцшильда). Поставленная им проблема о нахождении редукции на данные обратной задачи, отвечающей риччи-плоским метрикам, остается важной открытой проблемой. Им же с помощью нового и оригинального трюка - дифференииалъной редукиии - был выделен, на языке данных обратной задачи - функции $F$, класс плоских метрик с диагональной кривизной.

ТеОрема 3 [24]. Если в условиях теоремы 2 функиия F удовлетворяет соотношению

$$
\frac{\partial F_{i j}}{\partial s^{\prime}}\left(s, s^{\prime}, u\right)+\frac{\partial F_{j i}}{\partial s}\left(s^{\prime}, s, u\right)=0
$$

то $\widetilde{B}$ удовлетворяет уравнениям (15) и построенные по $\widetilde{B}$ коэффициенты вращения отвечают плоским метрикам вида (9), т.е. ортогональным криволинейным системам координат.

Система из уравнений (22) и (24) допускает следующее решение [24].

Пусть $\Phi_{i j}(x, y), i<j,-N(N-1) / 2$ произвольных функций от двух переменных и $\Phi_{i i}(x, y)-N$ произвольных антисимметричных функций:

$$
\Phi_{i i}(x, y)=-\Phi_{i i}(y, x)
$$


Положим

$$
\begin{gathered}
F_{i j}=\frac{\partial \Phi_{i j}\left(s-u^{i}, s^{\prime}-u^{j}\right)}{\partial s}, \quad F_{j i}=\frac{\partial \Phi_{i j}\left(s^{\prime}-u^{i}, s-u^{j}\right)}{\partial s}, \quad i \neq j, \\
F_{i i}=\frac{\partial \Phi_{i i}\left(s-u^{i}, s^{\prime}-u^{i}\right)}{\partial s} .
\end{gathered}
$$

Тогда $F=\left(F_{i j}\right)$ удовлетворяет уравнениям $(22)$ и $(24)$ и решение $K$ уравнения (21) с такой матрицей $F$ дает при любом фиксированном значении s коэфбициенты вращения ортогональной системы координат: $\beta_{i j}(u)=$ $K_{i j}(s, s, u)$. Заметим, что мы имеем $N(N+1) / 2$ функциональных параметров $\Phi_{i j}, i \leqslant j$, а общее решение зависит от $N(N-1) / 2$ функциональных параметров, поэтому этот метод дает эквивалентные классы одеваний.

Общая теория редукций, включая дифференциальные, которые могут понадобиться для выделения других классов метрик, обсуждается в [30]. В частности, с помощью некоторой вариации редукции (24) можно описать метрики с диагональной кривизной и постоянной секционной кривизной $K \neq 0$, т. е. ортогональные криволинейные координаты в пространствах постоянной секционной кривизны $K$ (В.Е. Захаров). В этом случае система (15) заменяется на уравнения

$$
\frac{\partial \beta_{i j}}{\partial u^{i}}+\frac{\partial \beta_{j i}}{\partial u^{j}}+\sum_{k \neq i, j} \beta_{k i} \beta_{k j}=-K H_{i} H_{j}, \quad i \neq j .
$$

\section{5. Конструкция Кричевера конечнозонных ортогональных кри-} волинейных координат. В [5] И. М. Кричевер предложил конечнозонную версию конструкции ортогональных криволинейных координат в евклидовых пространствах, которая, однако, в отличие от [24] не состоит из двух этапов: конструкции метрик с диагональной кривизной и последующего выделения среди них плоских метрик, а сразу дает указанные выше координаты.

Пусть Г - гладкая комплексная алгебраическая кривая, т. е. компактная гладкая риманова поверхность.

Выберем на Г три эффективных дивизора:

$$
P=P_{1}+\cdots+P_{N}, \quad D=\gamma_{1}+\cdots+\gamma_{g+l-1}, \quad R=R_{1}+\cdots+R_{l},
$$

где $g$ - род кривой $Г$ и $P_{i}, \gamma_{j}, R_{k} \in \Gamma$. В окрестности каждой точки $P_{i}, i=$ $1, \ldots, N$, выберем локальный параметр $k_{i}^{-1}$, обращающийся в нуль в указанной точке.

Функцией Бейкера-Ахиезера, отвечающей спектральным данным $S=\{\Gamma, P$, $D, R\}$, называется такая функция $\psi\left(u^{1}, \ldots, u^{N}, z\right), z \in \Gamma$, что:

1) $\psi \exp \left(-u^{i} k_{i}\right)$ аналитична в окрестности $P_{i}, i=1, \ldots, N$;

2) $\psi$ мероморфна на $\Gamma \backslash\left\{\bigcup P_{i}\right\}$ с полюсами в $\gamma_{j}, j=1, \ldots, g+l-1$;

3) $\psi\left(u, R_{k}\right)=1, k=1, \ldots, l$.

В случае дивизора $D$ общего положения такая функция существует и единственна и выражается через тэта-функцию кривой $Г$.

Если кривая $Г$ не связна, то предполагается, что ограничение функции Бейкера-Ахиезера на каждую связную компоненту удовлетворяет таким условиям. 
Возьмем дополнительный дивизор $Q=Q_{1}+\cdots+Q_{N}$ на $\Gamma$ такой, что $Q_{i} \in$ $\Gamma \backslash\{P \cup D \cup R\}, i=1, \ldots, N$, и положим

$$
x^{j}\left(u^{1}, \ldots, u^{N}\right)=\psi\left(u^{1}, \ldots, u^{N}, Q_{j}\right), \quad j=1, \ldots, N .
$$

Имеет место следующая теорема.

Теорема 4 [5]. 1) Пусть на Г задана такая голоморфная инволюция $\sigma$ : $\Gamma \rightarrow \Gamma$, umo

а) $\sigma$ имеет в точности $2 m$ неподвижных точек, $m \leqslant N \leqslant 2 m: P_{1}, \ldots, P_{N}$ и $2 m-N$ точек из $Q$;

б) $\sigma(Q)=Q$, m.е. инволючия либо переставляет точки из $Q$, либо оставляет их неподвижными:

$$
\sigma\left(Q_{k}\right)=Q_{\sigma(k)}, \quad k=1, \ldots, N
$$

в) $\sigma\left(k_{i}^{-1}\right)=-k_{i}^{-1}$ в окрестности $P_{i}, i=1, \ldots, N$;

г) существует такой мероморфньй дифференциал $\Omega$ на $\Gamma$, что его дивизоры нулей и полюсов имеют вид

$$
(\Omega)_{0}=D+\sigma D+P, \quad(\Omega)_{\infty}=R+\sigma R+Q
$$

д) Факторпространство $\Gamma_{0}=\Gamma / \sigma$ является гладкой алгебраической кривой.

Тогда $\Omega$ является поднятием некоторого мероморфного дифференииала $\Omega_{0}$ на $\Gamma_{0}$ и справедливы следующие равенства:

$$
\sum_{k, l} \eta_{k l} \frac{\partial x^{k}}{\partial u^{i}} \frac{\partial x^{l}}{\partial u^{j}}=\varepsilon_{i}^{2} H_{i}^{2}(u) \delta_{i j}
$$

где

$$
\begin{gathered}
H_{i}=\lim _{P \rightarrow P_{i}}\left(\psi e^{-u^{i} k_{i}}\right), \quad \eta_{k l}=\delta_{k, \sigma(l)} \operatorname{res}_{Q_{k}} \Omega_{0}, \\
\Omega_{0}=\frac{1}{2}\left(\varepsilon_{i}^{2} \lambda_{i}+O\left(\lambda_{i}\right)\right) d \lambda_{i}, \lambda_{i}=k_{i}^{-2}, \text { в точке } P_{i}, i=1, \ldots, N .
\end{gathered}
$$

2) Если, кроме того, существует такая антиголоморфная инволюиия $\tau$ : $\Gamma \rightarrow \Gamma$, что все неподвижные точки $\sigma$ неподвижны при $\tau$ и

$$
\tau^{*}(\Omega)=\bar{\Omega}
$$

то коэфбициенты $H_{i}(u)$ являются вещественными $п р и u^{1}, \ldots, u^{N} \in \mathbb{R}$ и $u^{1}, \ldots, u^{N}$ являются ортогональными криволинейными координатами $в$ плоском $N$-мерном пространстве с метрикой $\eta_{k l} d x^{k} d x^{l} 7$.

Если при этом все точки из $Q$ неподвижны под действием $\sigma u$

$$
\operatorname{res}_{Q_{1}} \Omega_{0}=\cdots=\operatorname{res}_{Q_{N}} \Omega_{0}=\eta_{0}^{2}>0,
$$

7 Легко заметить, что если существуют точки $Q_{k}$ и $Q_{l}, l=\sigma(k)$, которые переставляются инволюцией, то метрика $\eta$ является неопределенной. 
то функиии $x^{1}\left(u^{1}, \ldots, u^{N}\right), \ldots, x^{N}\left(u^{1}, \ldots, u^{N}\right)$ решают задачу погружения для ортогональных криволинейных координат $u^{1}, \ldots, u^{N}$ и метрики $d s^{2}=$ $H_{1}^{2}\left(d u^{1}\right)^{2}+\cdots+H_{N}^{2}\left(d u^{N}\right)^{2}$, гдe

$$
H_{i}=\frac{\varepsilon_{i} h_{i}}{\eta_{0}}, \quad i=1, \ldots, N .
$$

ЗАмЕчаниЕ 1. Теорема останется верной, если вместо а) мы предположим, что функции $\psi \exp \left(-f^{i}\left(u^{i}\right) k_{i}\right)$ аналитичны в окрестности $P_{i}$, где $f^{i}$ - некоторые функции одной переменной, обратимые в окрестности нуля, $i=1, \ldots, N$. Поэтому мы не будем различать ортогональные системы координат, которые получаются заменами вида

$$
u^{i} \rightarrow f^{i}\left(u^{i}\right) .
$$

Можно также заменить условие $\psi\left(u, R_{k}\right)=1, k=1, \ldots, l$, на

$$
\psi\left(u, R_{k}\right)=d_{k}, \quad k=1, \ldots, l,
$$

где все константы $d_{k}$ не обращаются в нуль. Более того, мы можем даже ограничиться предположением, что константы $d_{k}$ не обращаются одновременно в нуль:

$$
\left|d_{1}\right|^{2}+\cdots+\left|d_{l}\right|^{2} \neq 0
$$

и при этом теорема останется верной.

На случай ортогональных координат в пространствах постоянной кривизны метод Кричевера был недавно обобщен в [31].

\section{6. Системы координат, отвечающие сингулярным спектральным}

кривым. Конструкция Кричевера (теорема 4) приводит к тэта-функциональным формулам, которые сложны для их качественного анализа. В то же время при вырождении гладкой спектральной кривой Г мы можем получать более простые формулы, которые отвечают решениям, лежащим на границе пространства модулей, найденного Кричевером. Чтобы понять, как устроены конечнозонные ортогональные криволинейные координаты, также полезно найти среди них классические системы координат. Этому может служить следующая теорема.

ТЕОРема 5 [1]. Теорема 4 останется справедливой для сингулярной алгебрачческой кривой, если заменить $g$ на $p_{a}(\Gamma)$, т.е. на арифметический род $Г$, u предположение д) о гладкости кривой $\Gamma / \sigma$ заменить на условие, что $P_{1}, \ldots, P_{N}$ и полюсы $\Omega$ являются несингулярными точками.

Более того, мы можем предполагать, что ф удовлетворяет условиям (26) $u$ (27) вместо $\psi\left(u, R_{k}\right)=1, k=1, \ldots, l$.

В случае, когда $\Gamma_{\mathrm{nm}}$ есть объединение гладких рациональных кривых, т. е. $\mathbb{C} P^{1}$, процедура построения функций Бейкера-Ахиезера и ортогональных координат сводится к простым вычислениям с элементарными функциями и не идет дальше решения систем линейных уравнений. Мы продемонстрируем это на примерах. 
Отметим, что, оказывается, наиболее простые спектральные данные отвечают достаточно экзотическим координатам, в то время как для классических систем координат спектральные кривые устроены достаточно сложно.

Напомним, что регулярный дифференциал $\Omega$ на $\Gamma$ задается набором дифференциалов $\Omega_{1}, \ldots, \Omega_{s}$ на неприводимых компонентах $\Gamma_{1}, \ldots, \Gamma_{s}$. По его определению мы имеем $\sum_{i} \sum_{\pi^{-1}(P) \in \Gamma_{i}} \operatorname{res} \Omega_{i}=0$, где $\pi: \Gamma_{\mathrm{nm}} \rightarrow \Gamma-$ нормализация. Арифметический род $p_{a}$ - это размерность пространства регулярных дифференциалов и сумма берется по всем особым точкам $P$.

Если особенности состоят только из кратных точек, то эти дифференциалы могут иметь полюсы в точках пересечения компонент, причем если $P$ - точка пересечения компонент $\Gamma_{i_{1}}, \ldots, \Gamma_{i_{r}}$, то $\sum_{k=1}^{r} \operatorname{res}_{P} \Omega_{i_{k}}=0$. Арифметический род $p_{a}$ - это размерность пространства голоморфных регулярных дифференциалов, т. е. таких дифференциалов, что $\Omega_{j}$ могут иметь полюсы только в точках пересечения различных компонент и суммы вычетов в точках пересечения равны нулю.

ПримеР $5^{8}$. Рассмотрим простейшую сингулярную спектральную кривую: $\Gamma$ состоит из двух экземпляров $\mathbb{C} P^{1}$, т. е. из $\Gamma_{1}$ и $\Gamma_{2}$, которые пересекаются по двум точкам, что влечет $p_{a}(\Gamma)=1: a \sim b,(-a) \sim(-b),\{a,-a\} \subset \Gamma_{1}$, $\{b,-b\} \subset \Gamma_{2}$ (см. рис. 1). Рассмотрим случай, когда существенные особенности

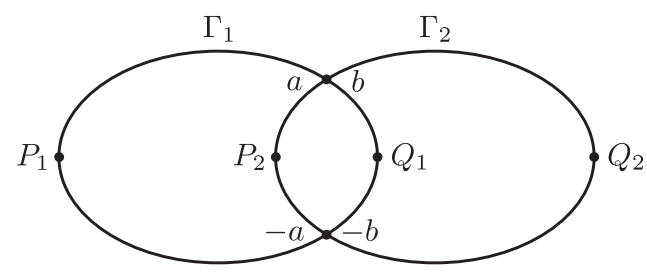

Рис. 1

лежат на различных неприводимых компонентах - в точках $P_{1}=\infty \in \Gamma_{1}$ и $P_{2}=\infty \in \Gamma_{2}$. Функция Бейкера-Ахиезера принимает вид

$$
\begin{array}{cc}
\psi_{1}\left(u^{1}, u^{2}, z_{1}\right)=e^{u^{1} z_{1}}\left(f_{0}\left(u^{1}, u^{2}\right)+\frac{f_{1}\left(u^{1}, u^{2}\right)}{z_{1}-\alpha_{1}}+\cdots+\frac{f_{k}\left(u^{1}, u^{2}\right)}{z_{1}-\alpha_{s_{1}}}\right), & z_{1} \in \Gamma_{1}, \\
\psi_{2}\left(u^{1}, u^{2}, z_{2}\right)=e^{u^{2} z_{2}}\left(g_{0}\left(u^{1}, u^{2}\right)+\frac{g_{1}\left(u^{1}, u^{2}\right)}{z_{2}-\beta_{1}}+\cdots+\frac{g_{n}\left(u^{1}, u^{2}\right)}{z_{2}-\beta_{s_{2}}}\right), & z_{2} \in \Gamma_{2} . \\
\psi_{1}(a)=\psi_{2}(b), \quad \psi_{1}(-a)=\psi_{2}(-b) . &
\end{array}
$$

Общее условие нормировки имеет вид

$$
\psi_{1}\left(R_{1, i}\right)=d_{1, i}, \quad \psi_{2}\left(R_{2, j}\right)=d_{2, j},
$$

\footnotetext{
8Этот простой пример мы изложим подробно, чтобы продемонстрировать процедуру построения; подробности других примеров можно найти в [1].
} 
где $R_{1, i} \in \Gamma_{1}, i=1, \ldots, l_{1}$, и $R_{2, j} \in \Gamma_{2}, j=1, \ldots, l_{2}$. Мы также имеем $l=$ $l_{1}+l_{2}=s_{1}+s_{2}$. Пусть

$$
\begin{aligned}
& \Omega_{1}=\frac{\left(z_{1}^{2}-\alpha_{1}^{2}\right) \cdots\left(z_{1}^{2}-\alpha_{l_{1}}^{2}\right)}{z_{1}\left(z_{1}^{2}-a^{2}\right)\left(z_{1}^{2}-R_{1,1}^{2}\right) \cdots\left(z_{1}^{2}-R_{1, l_{1}}^{2}\right)} d z_{1}, \\
& \Omega_{2}=\frac{\left(z_{2}^{2}-\beta_{1}^{2}\right) \cdots\left(z_{2}^{2}-\beta_{l_{2}}^{2}\right)}{z_{2}\left(z_{2}^{2}-b^{2}\right)\left(z_{2}^{2}-R_{2,1}^{2}\right) \cdots\left(z_{2}^{2}-R_{2, l_{2}}^{2}\right)} d z_{2} .
\end{aligned}
$$

Положим $Q_{1}=0 \in \Gamma_{1}, Q_{2}=0 \in \Gamma_{2}$. Если справедливы равенства

$$
\operatorname{res}_{a} \Omega_{1}=-\operatorname{res}_{b} \Omega_{2}, \quad \operatorname{res}_{-a} \Omega_{1}=-\operatorname{res}_{-b} \Omega_{2}, \quad \operatorname{res}_{Q_{1}} \Omega_{1}=\operatorname{res}_{Q_{2}} \Omega_{2},
$$

то дифференциал $\Omega$, заданный дифференциалами $\Omega_{1}$ и $\Omega_{2}$, является регулярным и условие (25) выполнено. В этом случае, согласно теореме 5 , координаты $u^{1}$ и $u^{2}$ такие, что

$$
x^{1}(u)=\psi_{1}(u, 0), \quad x^{2}(u)=\psi_{2}(u, 0),
$$

являются ортогональными.

Рассмотрим простейший случай $l_{1}=0$ и $l_{2}=1$. Тогда

$$
\psi_{1}=e^{u^{1} z_{1}} f_{0}\left(u^{1}, u^{2}\right), \quad \psi_{2}=e^{u^{2} z_{2}}\left(g_{0}\left(u^{1}, u^{2}\right)+\frac{g_{1}\left(u^{1}, u^{2}\right)}{z_{2}-c}\right) .
$$

Условие склейки в точках пересечения и условие нормировки принимают вид $\psi_{1}(a)=\psi_{2}(b), \psi_{1}(-a)=\psi_{2}(-b), \psi_{2}(r)=1, r=R \in \Gamma_{2}$, что влечет

$$
\begin{aligned}
& \psi_{1}=e^{u^{1} z_{1}} \frac{2 b(c-r) e^{a u^{1}+(b-r) u^{2}}}{(b+c)(b-r) e^{2 b u^{2}}-(b+r)(b-c) e^{2 a u^{1}}} \\
& \psi_{2}=e^{u^{2} z_{2}}\left(\frac{e^{-r u^{2}}\left((b-c) e^{2 a u^{1}}+(b+c) e^{2 b u^{2}}\right)(c-r)}{(b+c)(b-r) e^{2 b u^{2}}-(b-c)(b+r) e^{2 a u^{1}}}\right. \\
&\left.\quad+\frac{1}{z_{2}-c} \frac{\left(b^{2}-c^{2}\right)(r-c) e^{-r u^{2}}\left(e^{2 a u^{1}}-e^{2 b u^{2}}\right)}{(b+c)(r-b) e^{2 b u^{2}}+(b-c)(b+r) e^{2 a u^{1}}}\right) .
\end{aligned}
$$

Дифференциал $\Omega$ задается на компонентах дифференциалами

$$
\Omega_{1}=-\frac{d z_{1}}{z_{1}\left(z_{1}^{2}-a^{2}\right)}, \quad \Omega_{2}=-\frac{\left(z_{2}^{2}-c^{2}\right) d z_{2}}{z_{2}\left(z_{2}^{2}-b^{2}\right)\left(z_{2}^{2}-r^{2}\right)},
$$

и их вычеты в сингулярных точках равны $\operatorname{res}_{a} \Omega_{1}=\operatorname{res}_{-a} \Omega_{1}=-1 /\left(2 a^{2}\right)=$ $-\operatorname{res}_{b} \Omega_{2}=-$ res $_{-b} \Omega_{2}=\left(b^{2}-c^{2}\right) /\left(2 b^{2}\left(b^{2}-r^{2}\right)\right)$. Условие регулярности (25) принимает вид $\operatorname{res}_{Q_{1}} \Omega_{1}=1 / a^{2}=\operatorname{res}_{Q_{2}} \Omega_{2}=c^{2} /\left(r^{2} b^{2}\right)$, что влечет $a=b r / c$, $r=b / \sqrt{2+b^{2} / c^{2}}$. Непосредственными вычислениями получаем

$$
\left(x^{1}\right)^{2}+\left(x^{2}-e^{-r u^{2}} \frac{b(c-r)}{c\left(b^{2}-r^{2}\right)}\right)^{2}=e^{-2 r u^{2}} \frac{b^{2}(c-r)^{2}}{c^{2}\left(b^{2}-r^{2}\right)^{2}} .
$$

Координатные линии $u^{2}=$ const - это окружности с центрами на оси $x^{2}$. При $b= \pm 1$ эти окружности касаются оси $x^{1}$, а второе семейство координатных линий $u^{1}=$ const состоит из окружностей с центрами на оси $x^{1}$, которые касаются оси $x^{2}$ (см. рис. 2). 


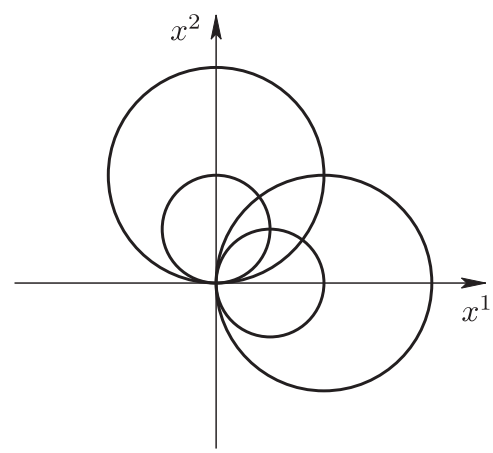

Рис. 2

Случай с той же спектральной кривой, но с существенными особенностями, расположенными на одной и той же компоненте, рассмотрен в [1].

ПримеР 6 (евклидовы координаты). Пусть Г есть объединение $n$ экземпляров $\Gamma_{1}, \ldots, \Gamma_{n}$ комплексной проективной прямой $\mathbb{C} P^{1}$. Положим

$$
P_{j}=\infty, \quad Q_{j}=0, \quad R_{j}=-1 \in \Gamma_{j}, \quad \psi_{j}\left(R_{j}\right)=1, \quad j=1, \ldots, N .
$$

На компонентах дифференциал $\Omega$ задается дифференциалами $\Omega_{j}=\frac{d z_{j}}{z_{j}\left(z_{j}^{2}-1\right)}$, а функция Бейкера-Ахиезера $\psi$ равна $\psi_{j}=e^{u^{j}} z_{j} f_{j}\left(u^{j}\right), j=1, \ldots, N$, и мы получаем евклидовы координаты в $\mathbb{R}^{N}: x^{j}=e^{u^{j}}$.

ПримеР 7 (полярные координаты). Кривая $\Gamma$ состоит из пяти неприводимых компонент $\Gamma_{1}, \ldots, \Gamma_{5}$, которые пересекаются как показано на рис. 3 . Мы имеем $p_{a}(\Gamma)=1$.

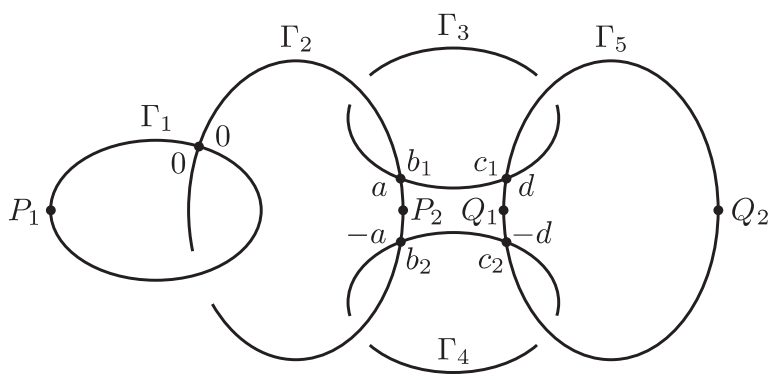

Рис. 3

Зададим инволюцию $\sigma$ на Г следующим образом:

а) на $\Gamma_{1}, \Gamma_{2}$ и $\Gamma_{3}$ инволюция имеет вид $\sigma\left(z_{j}\right)=-z_{j}$;

б) $\Gamma_{3}$ и $\Gamma_{4}$ переставляются под действием $\sigma$ и точки $b_{1}, c_{1}, \infty \in \Gamma_{3}$ отображаются в точки $b_{2}, c_{2}, \infty \in \Gamma_{4}$ соответственно. 
При специальном выборе дивизоров $D, P, Q$ и $R$ [1] полученные спектральные данные отвечают полярным координатам:

$$
x^{1}=\psi_{5}\left(Q_{1}\right)=r \cos \varphi, \quad x^{2}=\psi_{5}\left(Q_{2}\right)=r \sin \varphi, \quad r=e^{u^{1}}, \quad \varphi=u^{2} .
$$

ПримеР 8 (цилиндрические координаты). Кривая Г есть несвязное объединение кривой $\widehat{\Gamma}$ из предыдущего примера (полярные координаты) и копии $\Gamma_{6}$ кривой $\mathbb{C} P^{1}$. Все данные, относящиеся к $\widehat{\Gamma},-$ те же самые, что и в предыдущем примере. На $\Gamma_{6}$ возьмем $Q_{3}=0, P_{3}=\infty, R_{4}=-1$ и $\psi\left(R_{4}\right)=1$. Тогда мы имеем $\psi_{6}\left(u^{3}\right)=e^{u^{3}\left(z_{6}+1\right)}$ и

$$
x^{1}=\psi_{5}\left(Q_{1}\right)=r \cos \varphi, \quad x^{2}=\psi_{5}\left(Q_{2}\right)=r \sin \varphi, \quad x^{3}=\psi_{6}\left(Q_{3}\right)=z,
$$

где $r=e^{u^{1}}, \varphi=u^{2}$ и $z=u^{3}$.

Пример 9 (сферические координаты в $\mathbb{R}^{3}$ ). Кривая $Г$ состоит из 9 неприводимых компонент, которые пересекаются как показано на рис. 4. Мы имеем $p_{a}(\Gamma)=2$.

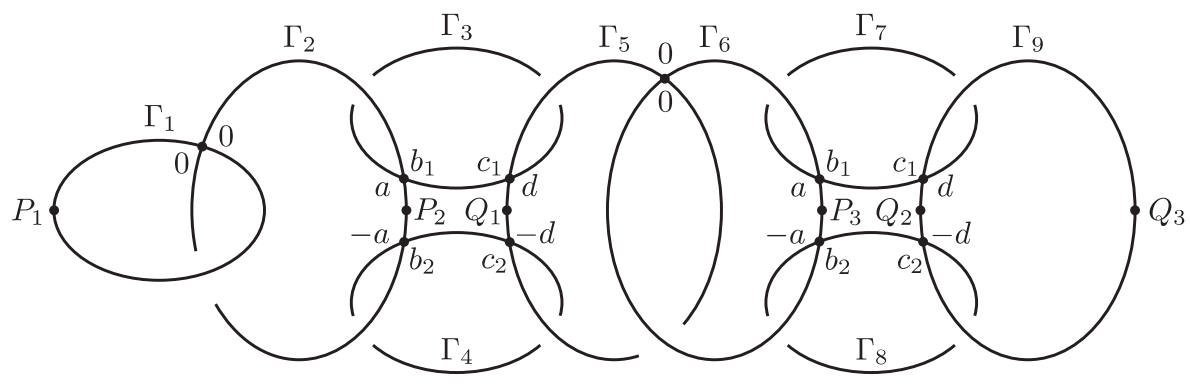

Рис. 4

При некотором выборе дивизоров $D, P, Q$ и $R$ (они указаны в [1]) эти спектральные данные приводят к сферическим координатам:

$$
\begin{aligned}
& x^{1}=\psi_{5}\left(Q_{1}\right)=r \sin \varphi, x^{2}=\psi_{9}\left(Q_{2}\right)=r \cos \varphi \sin \theta, \\
& x^{3}=\psi_{9}\left(Q_{3}\right)=r \cos \varphi \cos \theta, \quad r=e^{u^{1}}, \quad \varphi=u^{2}, \quad \theta=u^{3} .
\end{aligned}
$$

ПримеР 10 (сферические координаты в $\mathbb{R}^{N}$ ). Пусть $\Gamma^{(N-1)}-$ спектральная кривая и $\psi^{(N-1)}$ - функция Бейкера-Ахиезера для $(N-1)$-мерных сферических координат. Спектральная кривая $\Gamma^{(N)}$ для $N$-мерных сферических координат получается из $\Gamma^{(N-1)}$ и кривой, изображенной на рис. 5 , в результате их пересечения по точкам $0 \in \Gamma_{4 N-7} \subset \Gamma^{(N-1)}$ и $0 \in \Gamma_{4 N-6}$ (число неприводимых компонент $\Gamma^{(k)}$ равно $\left.4 k-3\right)$. Мы имеем $p_{a}\left(\Gamma^{(N)}\right)=N-1$.

Было бы интересно найти спектральные данные и для других известных ортогональных систем и, в частности, для эллиптических координат $u^{1}, u^{2}$, связанных с евклидовыми координатами $\left(x^{1}, x^{2}\right)$ соотношениями

$$
x^{1}=\cosh u^{1} \cos u^{2}, \quad x^{2}=\sinh u^{1} \sin u^{2} .
$$




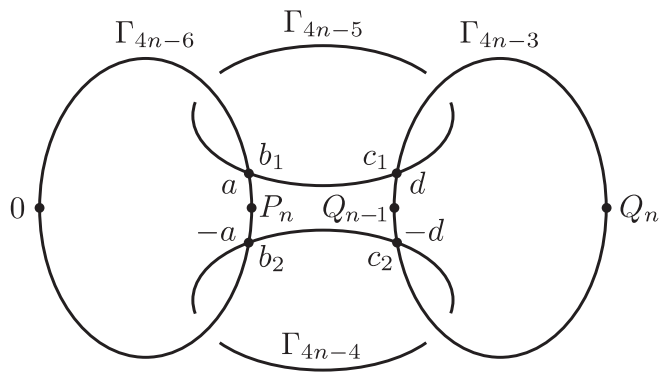

Рис. 5

2.7. Замечание о дискретных ортогональных координатах. В активно развивающейся сейчас дискретизации дифференциальной геометрии под системой дискретных координат в $\mathbb{R}^{N}$ понимается отображение

$$
x: \mathbb{Z}^{N} \rightarrow \mathbb{R}^{N}
$$

являющееся вложением решетки. Оператор сдвига $T_{i}$ вдоль $i$-й координаты действует на функциях $F: \mathbb{Z}^{N} \rightarrow \mathbb{R}^{k}$ по формуле

$$
T_{i} F\left(u^{1}, \ldots, u^{i-1}, u^{i}, u^{i+1}, \ldots, u^{N}\right)=F\left(u^{1}, \ldots, u^{i-1}, u^{i}+1, u^{i+1}, \ldots, u^{N}\right),
$$

и оператор частной производной $\Delta_{i}$ в $i$-м направлении определяется теперь как

$$
\Delta_{i} F(u)=T_{i} F(u)-F(u) .
$$

Следуя [32], говорят, что система координат является ортогональной, если выполняются следующие два условия:

1) (условие планарности) точки $\mathbf{x}(u), T_{i} \mathbf{x}(u), T_{j} \mathbf{x}(u), T_{i} T_{j} \mathbf{x}(u)$ лежат в одной плоскости для любой тройки $i, j, u$;

2) (условие вписанности) плоский четырехугольник, натянутый на точки $\mathbf{x}(u), T_{i} \mathbf{x}(u), T_{j} \mathbf{x}(u), T_{i} T_{j} \mathbf{x}(u)$, вписывается в окружность.

В [33] была получена процедура построения дискретных координат ДарбуЕгорова (плоских егоровских координат), основанная на формальной дискретизации функции Бейкера-Ахиезера для непрерывных координат Дарбу-Егорова из [5]. При этом дискретные координаты Дарбу-Егорова удовлетворяют свойству планарности, а свойство вписанности заменяется более сильным условием:

3) (дискретное условие Егорова) ребра решетки $X_{i}^{+}(u)=T_{i} \mathbf{x}(u)-\mathbf{x}(u)$ и $X_{j}^{-}(u)=T_{j}^{-1} \mathbf{x}(u)-\mathbf{x}(u)$ ортогональны для всех троек $i, j, u$ (отсюда следует то, что в каждом четырехугольнике из условия вписанности два противоположных угла являются прямыми и, следовательно, опираются на диаметр окружности, в которую вписывается четырехугольник).

Для дискретных координат Дарбу-Егорова существует дискретный потенциал - функция $\Phi$ такая, что $\Delta_{i} \Phi(u)=\left|T_{i} \mathbf{x}(u)-\mathbf{x}(u)\right|^{2}$.

Аналогично можно провести формальную дискретизацию (на уровне функций Бейкера-Ахиезера) координат, получаемых с помощью теоремы 5 . Поскольку в этом случае все вычисляется очень просто, мы можем явно найти 
примеры таких дискретных координат, которые хоть и удовлетворяют свойству планарности, но не удовлетворяют свойству вписанности. Это указывает на возникающие трудности при исследовании интересной проблемы: нахождении геометрически осмысленной дискретизации уравнений Ламе.

\section{3. Многообразия Фробениуса}

3.1. Уравнения ассоциативности и многообразия Фробениуса. Рассмотрим конечномерную алгебру с образующими $e_{1}, \ldots, e_{n}$ и коммутативным умножением

$$
e_{\alpha} \cdot e_{\beta}=c_{\alpha \beta}^{\gamma} e_{\gamma}, \quad c_{\alpha \beta \gamma}=\frac{\partial^{3} F(t)}{\partial t^{\alpha} \partial t^{\beta} \partial t^{\gamma}}, \quad c_{\alpha \beta}^{\gamma}=\eta^{\gamma \delta} c_{\alpha \beta \delta}, \quad \eta^{\alpha \beta}=\eta^{\beta \alpha}
$$

(где $F=F(t)$ - функция от $\left.t=\left(t^{1}, \ldots, t^{N}\right)\right)$. Алгебра является ассоциативной, т. е. выполняются соотношения

$$
\left(e_{\alpha} \cdot e_{\beta}\right) \cdot e_{\gamma}=e_{\alpha} \cdot\left(e_{\beta} \cdot e_{\gamma}\right) \text { для всех } \alpha, \beta, \gamma,
$$

тогда и только тогда, когда функция $F$ удовлетворяет уравнениям ассоииативности

$$
\frac{\partial^{3} F(t)}{\partial t^{\alpha} \partial t^{\beta} \partial t^{\lambda}} \eta^{\lambda \mu} \frac{\partial^{3} F(t)}{\partial t^{\gamma} \partial t^{\delta} \partial t^{\mu}}=\frac{\partial^{3} F(t)}{\partial t^{\gamma} \partial t^{\beta} \partial t^{\lambda}} \eta^{\lambda \mu} \frac{\partial^{3} F(t)}{\partial t^{\alpha} \partial t^{\delta} \partial t^{\mu}} .
$$

В действительности, при выполнении этих условий мы получаем целое семейство ассоциативных алгебр, зависящее от $N$-мерного параметра $t$.

Эти уравнения впервые возникли в квантовой теории поля, и вместе со следующими условиями:

1) $c_{1 \alpha \beta}=\eta_{\alpha \beta}, \alpha, \beta=1, \ldots, N$

2) $\operatorname{det}\left(\eta^{\alpha \beta}\right) \neq 0, \eta^{\alpha \beta} \eta_{\beta \gamma}=\delta_{\gamma}^{\alpha}$ и метрика $\eta_{\alpha \beta}-$ постоянная;

3) (условие квазиоднородности)

$$
F\left(\lambda^{d_{1}} t^{1}, \ldots, \lambda^{d_{N}} t^{N}\right)=\lambda^{d_{F}} F\left(t^{1}, \ldots, t^{N}\right),
$$

они образуют систему уравнений Виттена-Дийкграафа-Верлинде-Верлинде (WDVV) [34], [35].

Существует два обобщения условия квазиоднородности: предполагается, что существует векторное поле $E=\left(q_{\beta}^{\alpha} t^{\beta}+r^{\alpha}\right) \partial_{\alpha}$, которое удовлетворяет одному из следующих условий:

$\left.3^{\prime}\right)$ выполняется равенство

$$
E^{\alpha} \partial_{\alpha} F=d_{F} F
$$

В случае (29) поле $E$ имеет вид $E=d_{1} t^{1} \partial_{1}+\cdots+d_{N} t^{N} \partial_{n}$; это обобщение покрывает случай квантовых когомологий;

$3^{\prime \prime}$ ) так как, согласно [35], важно только, чтобы корреляторы $c_{i j k}$, т. е. третьи производные $F$, были квазиоднородны в смысле (29), достаточно потребовать, чтобы

$$
E^{\alpha} \partial_{\alpha} F=d_{F} F+\left(\text { полином второго порядка по } t^{1}, \ldots, t^{N}\right) \text {; }
$$


это обобщение важно для нас потому, что в наших примерах часть показателей $d_{i}$ равны -1 .

Геометрической формой решений уравнений WDVV является понятие фробениусова многообразия, введенное Б. А. Дубровиным [36]: многообразием Фробениуса называется область $U \subset \mathbb{R}^{N}$, оснащенная постоянной невырожденной метрикой $\eta_{\alpha \beta} d u^{\alpha} d u^{\beta}$ и решением уравнения ассоциативности (препотенциалом $F$, удовлетворяющим условиям 1$), 2$ ) и наиболее общему условию квазиоднородности $3^{\prime \prime}$ ).

Требование квазиоднородности пришло из физики, и кроме квантовой теории поля фробениусовы многообразия играют важную роль в теории изомонодромных деформаций [37 ${ }^{9}$. Однако это требование является и наиболее ограничительным. Поэтому в современной фробениусовой геометрии оно часто опускается.

До [2] все известные фробениусовы многообразия исчерпывались примерами Дубровина фробениусовых структур на пространствах орбит групп Кокстера (здесь роль плоской метрики играет метрика Сато; решения уравнений WDVV, отвечающие особенностям типа $A_{n}$, были найдены в [35]) и на пространствах Гурвица, квантовыми когомологиями и расширенными пространствами модулей комплексных структур на многообразиях Калаби-Яо [38], а также "удвоениями" пространств Гурвица, найденными В. Шрамченко [39].

В каждом из этих примеров многообразие Фробениуса имеет специальный геометрический смысл. Примеры из [2] получены аналитическими методами (с помощью конечнозонного интегрирования), являются алгебраическими в том смысле, что корреляторы $c_{i j k}=\frac{\partial^{3} F}{\partial x^{i} \partial x^{j} \partial x^{k}}$ являются алгебраическими функциями, и не являются полупростыми.

3.2. Конечнозонные многообразия Фробениуса. Существует важная связь между решениями уравнений ассоциативности и плоскими егоровскими метриками, открытая Дубровиным [40]. А именно, пусть

$$
\eta_{\alpha \beta} d x^{\alpha} d x^{\beta}=\sum_{i=1}^{N} H_{i}^{2}(u)\left(d u^{i}\right)^{2}
$$

- плоская егоровская метрика и $x^{1}, \ldots, x^{N}$ - координаты в некоторой области, в которой коэффициенты $\eta_{\alpha \beta}$ постоянны. Мы имеем

$$
\eta^{\alpha \beta}=\sum_{i=1}^{N} H_{i}^{-2} \frac{\partial x^{\alpha}}{\partial u^{i}} \frac{\partial x^{\beta}}{\partial u^{i}},
$$

и условия симметричности коэффициентов вращения и нулевой кривизны влекут существование такой функции $F(t)$, что

$$
c_{\alpha \beta \gamma}=\sum_{i=1}^{N} H_{i}^{2} \frac{\partial u^{i}}{\partial x^{\alpha}} \frac{\partial u^{i}}{\partial x^{\beta}} \frac{\partial u^{i}}{\partial x^{\gamma}}=\frac{\partial^{3} F}{\partial x^{\alpha} \partial x^{\beta} \partial x^{\gamma}}
$$

${ }^{9}$ Для изомонодромных деформаций представляют интерес только полупростые многообразия, т. е. не содержащие нильпотентных элементов. 
и выполняются уравнения ассоциативности

$$
c_{\alpha \beta}^{\lambda} c_{\lambda \gamma}^{\mu}=c_{\alpha \lambda}^{\mu} c_{\beta \gamma}^{\lambda} \quad \text { для всех } \alpha, \beta, \gamma=1, \ldots, N,
$$

где

$$
c_{\beta \gamma}^{\alpha}=\sum_{i} \frac{\partial x^{\alpha}}{\partial u^{i}} \frac{\partial u^{i}}{\partial x^{\beta}} \frac{\partial u^{i}}{\partial x^{\gamma}} .
$$

При условии, что ассоциативная алгебра полупроста, обратное также верно: можно построить по решению $F(t)$ уравнений ассоциативности егоровскую метрику, удовлетворяющую (31).

Поскольку $u^{1}, \ldots, u^{N}$ являются ортогональными криволинейными координатами в плоском пространстве, они могут получаться методом Кричевера (см. теорему 4). В его работе [5] указаны дополнительные ограничения на спектральные данные, которые приводят к егоровским метрикам и, тем самым, решениям уравнений ассоциативности. Однако из свойств тэта-функций, повидимому, можно вывести, что полученные явные тэта-функциональные формулы не дают квазиоднородных решений.

В то же время можно ожидать, что решения, выраженные в элементарных функциях и отвечающие сингулярным спектральным кривым, могут быть квазиоднородными и задавать многообразия Фробениуса. Это и было показано в работе [2].

Следующая теорема [2] выделяет специальный случай, когда конструкция теоремы 5 приводит к плоским егоровским метрикам и квазиоднородным решениям уравнений ассоциативности.

ТЕОремА 6. 1) Пусть выполняются условия теоремь 5 и Г-спектральная кривая, все неприводимые компоненты которой являются рациональными кривыми (комплексными проективными прямыми $\mathbb{C} P^{1}$ ).

Въделим на каждой компоненте $\Gamma_{i}$ nару точек $P_{i}=\infty, Q_{i}=0$ и глобальный параметр $k_{i}^{-1}=z_{i}, i=1, \ldots, N$. Предположим, что все сингулярные точки - это двукратные точки пересечения различных компонент и каждая такая точка пересечения $a \in \Gamma_{i} \cap \Gamma_{j}$ имеет одинаковые координаты на обеих компонентах:

$$
z_{i}(a)=z_{j}(a)
$$

а инволюиия $\sigma$ на каждой компоненте имеет вид

$$
\sigma\left(z_{i}\right)=-z_{i}
$$

Тогда метрика

$$
d s^{2}=\eta_{k l} d x^{k} d x^{l}=\sum_{i} H_{i}^{2}\left(d u^{i}\right)^{2}, \quad H_{i}=H_{i}\left(u^{1}, \ldots, u^{N}\right), \quad i=1, \ldots, N,
$$

построенная по этим спектралъным даннъм, является плоской егоровской метрикой (метрикой Дарбу-Егорова).

2) Предположим также, что спектральная кривая связна и функиия Бейкера-Ахиезера нормирована в одной точке $r$ :

$$
\psi(u, r)=1, \quad R=r \in \Gamma
$$


Тогда функиии

$$
c_{\alpha \beta \gamma}(x)=\sum_{i=1}^{n} H_{i}^{2} \frac{\partial u^{i}}{\partial x^{\alpha}} \frac{\partial u^{i}}{\partial x^{\beta}} \frac{\partial u^{i}}{\partial x^{\gamma}}
$$

являются однородными:

$$
c_{\alpha \beta \gamma}\left(\lambda x^{1}, \ldots, \lambda x^{n}\right)=\frac{1}{\lambda} c_{\alpha \beta \gamma}\left(x^{1}, \ldots, x^{n}\right) .
$$

Чтобы из найденного решения уравнений ассоциативности получить многообразие Фробениуса, надо добавить к образующим $e_{1}, \ldots, e_{N}$ ассоциативной алгебры единицу $e_{0}$ и нильпотентный элемент $e_{N+1}$. Такое расширение дается следующей алгебраической леммой.

ЛЕмма 1 [2]. Пусть $F\left(t^{1}, \ldots, t^{N}\right)$ - решение уравнений ассочиативности с постоянной метрикой $\eta_{\alpha \beta}$. Тогда функиия

$$
\widetilde{F}\left(t^{0}, t^{1}, \ldots, t^{n}, t^{N+1}\right)=\frac{1}{2}\left(\eta_{\alpha \beta} t^{\alpha} t^{\beta} t^{0}+\left(t^{0}\right)^{2} t^{N+1}\right)+F\left(t^{1}, \ldots, t^{N}\right)
$$

удовлетворяет уравнениям ассоциативности (28) с метрикой

$$
\widetilde{\eta}=\left(\begin{array}{lll}
0 & 0 & 1 \\
0 & \eta & 0 \\
1 & 0 & 0
\end{array}\right)
$$

и ассочиативная алгебра, порожденная элементами $e_{0}, e_{1}, \ldots, e_{N}, e_{N+1}, c$ законом умножения

$$
e_{i} \cdot e_{j}=c_{i j}^{k} e_{k}, \quad c_{i j}^{k}=\tilde{\eta}^{k l} \frac{\partial^{3} \widetilde{F}}{\partial t^{l} \partial t^{i} \partial t^{j}},
$$

имеет единицу $e_{0}: e_{o} \cdot e_{k}=e_{k}$ для всех $k=0, \ldots, n+1$, и нильпотентньй элемент $e_{N+1}: e_{N+1}^{2}=0$. Более того, если $F$ квазиоднородна $u d_{\alpha}+d_{\beta}=c$ для всех $\alpha, \beta$ таких, что $\eta_{\alpha \beta} \neq 0$, то $\widetilde{F}$ также квазиоднородна $c d_{0}=d_{F}-c$, $d_{N+1}=2 c-d_{F}$ и теми же значениями $d_{\alpha}, \alpha=1, \ldots, N$, что и для $F$.

Приведем два простейших примера фробениусовых многообразий, получаемых с помощью теоремы 6.

Заметим, что утверждение 1) этой теоремы дает решения уравнения ассоциативности, а утверждение 2) выделяет среди них некоторый подкласс квазиоднородных решений: существуют квазиоднородные решения, чьи спектральные данные не удовлетворяют достаточным условиям из 2). Пример 11 покрывается условием 2), а пример 12 - нет.

ПримеР 11. Пусть Г образована двумя комплексными проективными прямыми $\Gamma_{1}$ и $\Gamma_{2}$, которые пересекаются по паре точек (см. рис. 6$):\left\{a,-a \in \Gamma_{1}\right\} \sim$ $\left\{a,-a \in \Gamma_{2}\right\}$. 


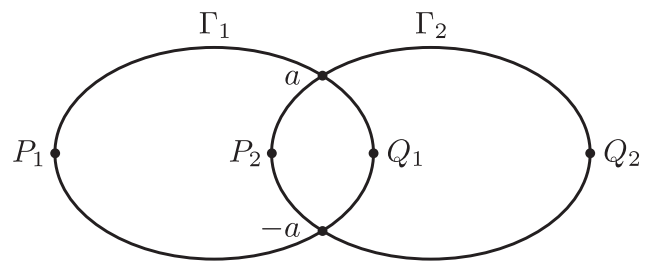

Рис. 6

Пусть $N=2, l=1$ и функция Бейкера-Ахиезера $\psi$ нормирована условием $\psi_{2}(r)=1$ в точке $r \in \Gamma_{2}$. Препотенциал принимает вид

$$
\begin{aligned}
& F_{a, c}\left(x^{1}, x^{2}\right)=\frac{1}{4 a c}\left(2 x_{2} \sqrt{\left(a^{2}-c^{2}\right) x_{1}^{2}+c^{2} x_{2}^{2}}\right. \\
& \quad+2 c x_{1}^{2} \log \left(-\frac{c x_{2}+\sqrt{\left(a^{2}-c^{2}\right) x_{1}^{2}+c^{2} x_{2}^{2}}}{x_{1}}\right)-\sqrt{2 c^{2}-a^{2}}\left(x_{1}^{2}+x_{2}^{2}\right) \\
& \left.\quad \times \log \left(c^{2}\left(x_{1}^{2}-3 x_{2}^{2}\right)+a^{2}\left(x_{2}^{2}-x_{1}^{2}\right)-2 x_{2} \sqrt{2 c^{2}-a^{2}} \sqrt{\left(a^{2}-c^{2}\right) x_{1}^{2}+c^{2} x_{2}^{2}}\right)\right)
\end{aligned}
$$

и удовлетворяет уравнениям ассоциативности при $\eta_{\alpha \beta}=\delta_{\alpha \beta}$. Он зависит от двух дополнительных параметров $a$ и $c$, и при $a=1, c=2 / \sqrt{7}$ формулы для координат и корреляторов являются достаточно простыми:

$$
\begin{gathered}
x^{1}=\frac{4(7-\sqrt{7}) e^{u^{1}-u^{2}}}{(21-6 \sqrt{7}) e^{2 u^{1}}+(7+2 \sqrt{7}) e^{2 u^{2}}}, \\
x^{2}=\frac{e^{-2 u^{2}}\left(3(\sqrt{7}-3) e^{2 u^{1}}+(5+\sqrt{7}) e^{2 u^{2}}\right)}{3(\sqrt{7}-2) e^{2 u^{1}}+(2+\sqrt{7}) e^{2 u^{2}}}, \\
c_{111}=-\frac{9 x_{1}^{8}+51 x_{1}^{6} x_{2}^{2}+88 x_{1}^{4} x_{2}^{4}+\left(2 x_{1}^{2} x_{2}^{3}+4 x_{2}^{5}\right) \sqrt{\left(3 x_{1}^{2}+4 x_{2}^{2}\right)^{3}}+48 x_{1}^{2} x_{2}^{6}}{2 x_{1}\left(3 x_{1}^{4}+7 x_{1}^{2} x_{2}^{2}+4 x_{2}^{4}\right)^{2}}, \\
c_{112}=\frac{9 x_{1}^{6} x_{2}+15 x_{1}^{4} x_{2}^{3}-8 x_{1}^{2} x_{2}^{5}+\left(2 x_{1}^{2} x_{2}^{2}+4 x_{2}^{4}\right) \sqrt{\left(3 x_{1}^{2}+4 x_{2}^{2}\right)^{3}}-16 x_{2}^{7}}{2\left(3 x_{1}^{4}+7 x_{1}^{2} x_{2}^{2}+4 x_{2}^{4}\right)^{2}}, \\
c_{122}=-\frac{9 x_{1}^{7}+15 x_{1}^{5} x_{2}^{2}-8 x_{1}^{3} x_{2}^{4}+\left(2 x_{1}^{3} x_{2}+4 x_{1} x_{2}^{3}\right) \sqrt{\left(3 x_{1}^{2}+4 x_{2}^{2}\right)^{3}}-16 x_{1} x_{2}^{6}}{2\left(3 x_{1}^{4}+7 x_{1}^{2} x_{2}^{2}+4 x_{2}^{4}\right)^{2}}, \\
c_{222}=\frac{-27 x_{1}^{6} x_{2}-16 x_{2}^{7}-72 x_{1}^{2} x_{2}^{5}+\left(4 x_{1}^{2} x_{2}^{2}+2 x_{1}^{4}\right) \sqrt{\left(3 x_{1}^{2}+4 x_{2}^{2}\right)^{3}}-81 x_{1}^{4} x_{2}^{3}}{2\left(3 x_{1}^{4}+7 x_{1}^{2} x_{2}^{2}+4 x_{2}^{4}\right)^{2}} .
\end{gathered}
$$

ПримеР 12. Пусть комплексная кривая $Г$ - такая же, как в примере 11. В отличие от примера 11 мы предположим, что

$$
P_{1}=\infty \in \Gamma_{1}, \quad P_{2}=0 \in \Gamma_{1}, \quad Q_{1}=\infty \in \Gamma_{2}, \quad Q_{2}=0 \in \Gamma_{2},
$$

точка нормировки $R=r$ лежит на $\Gamma_{1}$ и дивизор полюсов $D=c$ лежит на $\Gamma_{2}$ (см. рис. 7). Но мы не предполагаем, что точки пересечения имеют одинаковые координаты (предположения утверждения 2) теоремы 6 не выполнены):

$$
a \sim b, \quad-a \sim-b, \quad \pm a \in \Gamma_{1}, \quad \pm b \in \Gamma_{2}, \quad a \neq b .
$$




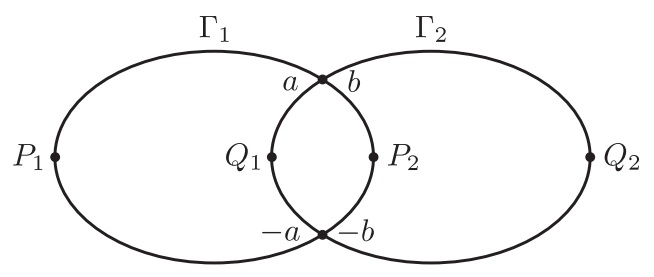

Рис. 7

Препотенциал $F\left(x^{1}, x^{2}\right)$ имеет вид

$$
F\left(x^{1}, x^{2}\right)=-\frac{1}{8}\left(\left(x^{1}\right)^{2}+\left(x^{2}\right)^{2}\right) \log \left(\left(x^{1}\right)^{2}+\left(x^{2}\right)^{2}\right)
$$

и включается в линейное семейство квазиоднородных функций

$$
\begin{aligned}
F_{q}\left(x^{1}, x^{2}\right)= & \\
& \left(\left(x^{1}\right)^{2}+\left(x^{2}\right)^{2}\right) \arctan \left(\frac{x^{1}}{x^{2}}\right) \\
& -\frac{1}{8}\left(\left(x^{1}\right)^{2}+\left(x^{2}\right)^{2}\right) \log \left(\left(x^{1}\right)^{2}+\left(x^{2}\right)^{2}\right), \quad q \in \mathbb{R},
\end{aligned}
$$

которые удовлетворяют уравнениям ассоциативности при $\eta_{\alpha \beta}=\delta_{\alpha \beta}$.

Корреляторы для $F$ (т. е. $q=0)$ имеют наиболее простой вид:

$$
\begin{aligned}
& c_{111}=-\frac{3}{2} \frac{x^{1}}{\left(x^{1}\right)^{2}+\left(x^{2}\right)^{2}}+\frac{\left(x^{1}\right)^{3}}{\left(\left(x^{1}\right)^{2}+\left(x^{2}\right)^{2}\right)^{2}}, \\
& c_{112}=-\frac{1}{2} \frac{x^{2}}{\left(x^{1}\right)^{2}+\left(x^{2}\right)^{2}}+\frac{\left(x^{1}\right)^{2} x^{2}}{\left(\left(x^{1}\right)^{2}+\left(x^{2}\right)^{2}\right)^{2}},
\end{aligned}
$$

и формулы для $c_{122}$ и $c_{222}$ получаются из предыдущих перестановкой индексов $1 \leftrightarrow 2$.

Примеры 11 и 12 описывают квазиоднородные деформации кольца когомологий $\mathbb{C} P^{2} \sharp \mathbb{C} P^{2}$. В самом деле, имеются стандартные образующие $e_{0}, \ldots, e_{3}$ в $H^{*}\left(\mathbb{C} P^{2} \sharp \mathbb{C} P^{2} ; \mathbb{C}\right)$ :

$$
e_{0} \in H^{0}, \quad e_{1}, e_{2} \in H^{2}, \quad e_{3} \in H^{4}, \quad e_{1}^{2}=e_{2}^{2}=e_{3}, \quad e_{1} e_{2}=0 .
$$

Справедливо тождество $d_{i}=\left(\operatorname{deg} e_{i}\right) / 2$. Эти деформации изменяют правила умножения для двумерных классов посредством добавления двумерных членов:

$$
e_{i} e_{j}=e_{3}+c_{i j}^{k}(t) e_{k}, \quad 1 \leqslant i, j \leqslant 2 .
$$

\section{4. Солитонные уравнения с самосогласованными источниками и отвечающие им деформации спектральных кривых}

4.1. Уравнения с самосогласованными источниками. В отличие от $(1+1)$-мерных солитонных уравнений, которые имеют представление Лакса

$$
L_{t}=[L, A]
$$


уравнения с самосогласованными источниками представляются в виде

$$
L_{t}=[L, A]+C,
$$

где $C$ - выражения от решений $\psi_{k}$ линейных задач $L \psi_{k}=\lambda_{k} \psi_{k}, k=1, \ldots, l$. $\mathrm{K}$ уравнениям с самосогласованными источниками применим метод обратной задачи и, по-видимому, с этой формально алгебраической точки зрения они впервые были выведены В. К. Мельниковым [41]. В физической литературе они впервые появились в работе В. Е. Захарова и Е. А. Кузнецова [42], в частности, объяснивших физический смысл уравнений Кортевега-де Фриза и КадомцеваПетвиашвили

$$
3 u_{y y}-\frac{\partial}{\partial x}\left(u_{t}+6 u u_{x}+u_{x x x}\right)=3 \frac{\partial^{2}}{\partial x^{2}}|\psi|^{2}, \quad i \psi_{t}=\psi_{x x}+u \psi
$$

с самосогласованными источниками.

Укажем два важных обстоятельства:

1) каждое такое уравнение имеет в качестве предшественника солитонное уравнение $L_{t}=[L, A]$;

2) для корректной постановки задачи надо нормировать функции $\psi$ (они должны отвечать тем или иным фиксированным спектральным характеристикам) и иметь выражение $C$, попадающее в класс рассматриваемых решений например, быстро убывающих или периодических.

Решения этих уравнений обладают интересными качественными свойствами: например, становятся возможными рождение и аннигиляция солитонов [43]. Мы обсудим это в п. 4.3.

В периодическом случае оператору $L$ отвечает спектральная кривая, которая, как оказалось, может деформироваться под действием уравнения с самосогласованными источниками, причем деформация состоит в появлении или исчезновении особых точек. Это впервые было обнаружено в [3] на примере конформного потока, возникающего в дифференциальной геометрии поверхностей, и затем изучено для уравнения КП с самосогласованными источниками [4]. Эти два факта и будут изложены ниже в пп. 4.2 и 4.3 соответственно.

\section{2. Спектральные кривые погруженных торов и конформный по-} ток. В теории представления Вейерштрасса погруженных поверхностей в $\mathbb{R}^{3}$ и $\mathbb{R}^{4}$ (см. обзор [13]) поверхности описываются в терминах решений уравнения $\mathscr{D} \psi=0$ в случае поверхностей в $\mathbb{R}^{3}$ и $\mathscr{D} \psi=\mathscr{D}^{\vee} \varphi=0$ в случае поверхностей в $\mathbb{R}^{4}$, где

$$
\mathscr{D}=\left(\begin{array}{cc}
0 & \partial \\
-\bar{\partial} & 0
\end{array}\right)+\left(\begin{array}{cc}
U & 0 \\
0 & \bar{U}
\end{array}\right), \quad \mathscr{D}^{\vee}=\left(\begin{array}{cc}
0 & \partial \\
-\bar{\partial} & 0
\end{array}\right)+\left(\begin{array}{cc}
\bar{U} & 0 \\
0 & U
\end{array}\right) .
$$

В случае поверхностей в $\mathbb{R}^{3}$ потенциал $U$ является вещественнозначным и $\mathscr{D}=\mathscr{D}^{\vee}$.

Для торов эти операторы имеют двоякопериодические коэффициенты и естественно рассмотреть их спектральные кривые на нулевом уровне энергии. Как оказалось, эти спектральные кривые содержат в себе информацию о значениях функционала Уиллмора, который определяется как

$$
\mathcal{W}(M)=\int_{M}|\mathbf{H}|^{2} d \mu
$$


где $\mathbf{H}$ - вектор средней кривизны и $d \mu$ - форма объема на поверхности $M$, индуцированная погружением. Этот функционал конформно инвариантен в том смысле, что

$$
\mathcal{W}(M)=\mathcal{W}(f(M))
$$

где $M$ - замкнутая поверхность и $f: S^{k} \rightarrow S^{k}$ - конформное преобразование $S^{k}=\mathbb{R}^{k} \cup\{\infty\}, k \geqslant 3$, такое, что $M \subset \mathbb{R}^{k}$ и $f(M) \subset \mathbb{R}^{k}$.

Для торов вращения потенциал $U$ зависит от одной переменной и мы получаем редукцию задачи $\mathscr{D} \psi=0$ к спектральной задаче Захарова-Шабата для одномерного оператора Дирака. При этом функционал Уиллмора $\mathcal{W}$ является первым интегралом Крускала-Миуры, связанным с этим оператором иерархий модифицированных уравнений КдФ. Другие интегралы Крускала-Миуры иерархии модифицированных уравнений КдФ обобщаются до (нелокальных) законов сохранения иерархий, связанных с двумерным оператором $\mathscr{D}$ (иерархий модифицированных уравнений Веселова-Новикова и Дэви-Стюартсона).

Это приводит нас к наблюдению о наличии связи между геометрией поверхности и спектральными свойствами оператора $\mathscr{D}$, входящего в ее представление Вейерштрасса [15].

Предложенный нами подход к доказательству гипотезы Уиллмора, основанный на этой связи, пока не привел к успеху.

Другое же наше предположение о том, что высшие двумеризованные интегралы Крускала-Миуры, а с ними и вся спектральная кривая, конформно инвариантны, было подтверждено в [44]. Для этого был использован конформный поток, предложенный П. Г. Гриневичем.

Поскольку группа конформных преобразований пространства $\mathbb{R}^{k} \cup\{\infty\}$ при $k \geqslant 3$ порождается трансляциями, растяжениями и инверсиями, а потенциал $U$ инвариантен относительно трансляций и растяжений, то инвариантность спектральной кривой достаточно доказать для инверсий. Для этого рассмотрим генератор инверсий в группе конформных преобразований и выпишем его действие на потенциал. Оно имеет вид

$$
U_{t}=\left|\psi_{2}\right|^{2}-\left|\psi_{1}\right|^{2}
$$

где тор в $\mathbb{R}^{3}$ задается по решению $\psi=\left(\psi_{1}, \psi_{2}\right)^{\top}$ уравнения $\mathscr{D} \psi=0$.

Предложение 1 [44]. Все мулътипликаторы функций Флоке оператора $\mathscr{D}$ сохраняются при действии потока (32), отвечающего инверсии тора в $\mathbb{R}^{3}$, а следовательно, они сохраняются при инверсии.

В [3] рассмотрена аналогичная задача для торов в $\mathbb{R}^{4}$ и показано, что следующему генератору инверсий:

$$
\begin{gathered}
\partial_{t} x^{1}=2 x^{1} x^{3}, \quad \partial_{t} x^{2}=2 x^{2} x^{3}, \\
\partial_{t} x^{3}=\left(x^{3}\right)^{2}-\left(x^{1}\right)^{2}-\left(x^{2}\right)^{2}-\left(x^{4}\right)^{2}, \quad \partial_{t} x^{4}=2 x^{4} x^{3},
\end{gathered}
$$

отвечает деформация потенциала $U$ вида

$$
\partial_{t} U=\varphi_{1} \bar{\psi}_{1}-\bar{\varphi}_{2} \psi_{2}, \quad \partial_{t} \bar{U}=\bar{\varphi}_{1} \psi_{1}-\varphi_{2} \bar{\psi}_{2}, \quad \mathscr{D} \psi=\mathscr{D}^{\vee} \varphi=0,
$$


где $\psi=\left(\psi_{1}, \psi_{2}\right)^{\top}$ и $\varphi=\left(\varphi_{1}, \varphi_{2}\right)^{\top}$ определяют тор в $\mathbb{R}^{4}$ посредством представления Вейерштрасса. Аналогично трехмерному случаю было показано, что справедливо следующее утверждение.

ПреДЛОжениЕ 2 [3]. Все мулътипликаторы функиий Флоке оператора $\mathscr{D}$ сохраняются под действием преобразования потенииала $U$ вида (33), где бункции $\psi$ и являются решениями уравнений $\mathscr{D} \psi=\mathscr{D}^{\vee} \varphi=0$ и квадратичные выражения $\psi_{i}^{2}, \varphi_{j}^{2}, i j,=1,2$, двоякопериодичны.

Заметим, что уравнения (32) и (33) являются уравнениями с самосогласованными источниками, которые при отсутствии этих источников переходят в стационарное уравнение $U_{t}=0$.

В то же время были известны явно просчитанные примеры спектральных кривых тора Клиффорда

$$
x_{1}^{2}+x_{2}^{2}=x_{3}^{2}+x_{4}^{2}=\frac{1}{2}
$$

в трехмерной сфере $S^{3} \subset \mathbb{R}^{4}$ и его стереографической проекции в $\mathbb{R}^{3}$. Гипотеза Уиллмора состоит в том, что на торе Клиффорда функционал Уиллмора достигает своего минимального значения (среди всех погруженных торов), оно равно $2 \pi^{2}$.

Для тора Клиффорда в $S^{3} \subset \mathbb{R}^{4}$ спектральная кривая является комплексной проективной прямой: $\Gamma=\mathbb{C} P^{1}$, а для его проекции в $\mathbb{R}^{3}$ - это рациональная кривая с двумя двойными точками [45]. Подробный анализ потока (33) привел к следующему результату.

Теорема 7 [3]. Эволюиионный поток (33), действующий на двоякопериодических потенииалах, сохраняет мультипликаторы Флоке оператора $\mathscr{D}$, но может деформировать его спектральную кривую (на нулевом уровне энергии), причем деформация сводится $к$ склейке и расклейке двойных точек.

Поскольку высшие законы сохранения для иерархий модифицированных уравнений Веселова-Новикова и Дэви-Стюартсона выражаются через асимптотическое поведение мультипликаторов, то они являются и законами сохранения для потока (33).

4.3. Конечнозонные решения уравнений КдФ и КП с самосогласованными источниками. Пусть $\Gamma$ - риманова поверхность рода $g$ (спектральная кривая), на которой выделена точка $P$ с заданным около нее локальным параметром $k^{-1}, k(P)=\infty$, и на которой задан дивизор (общего положения) $D=\gamma_{1}+\cdots+\gamma_{g}, \gamma_{i} \in \Gamma, i=1, \ldots, N$. Предположим также, что на $\Gamma$ заданы $2 N$ попарно различных отмеченных точек $R_{l}^{ \pm}, l=1, \ldots, N$, которые также отличны от $P$ и всех точек из $D$.

Согласно теории функций Бейкера-Ахиезера существует единственная функция $\psi(\gamma, x, y, t, \tau), \tau=\left(\tau_{1}, \ldots, \tau_{N}\right), \gamma \in \Gamma$, такая, что

1) $\psi$ мероморфна по $\gamma$ на $\Gamma \backslash P$ и имеет $g+N$ простых полюсов в точках $\gamma_{1}, \ldots, \gamma_{g}, R_{1}^{+}, \ldots, R_{N}^{+}$

$2)\left.\operatorname{res} \psi(\lambda, x, y, t, \tau)\right|_{\lambda=R_{l}^{+}}=\tau_{l} \psi\left(R_{l}^{-}, x, y, t, \tau\right), l=1, \ldots, N$; 
3) $\psi$ имеет существенную особенность в точке $P$ и

$$
\psi(\gamma, x, y, t, \tau)=e^{k x+k^{2} y+k^{3} t}\left(1+\sum_{m>0} \frac{\xi_{m}(x, y, t, \tau)}{k^{m}}\right) \quad \text { при } \gamma \rightarrow P .
$$

Кроме того, существует единственная сопряженная 1-форма Бейкера-Ахиезера ${ }^{10} \psi^{*}(\gamma, x, y, t, \tau)$ со следующими свойствами:

$\left.1^{*}\right) \psi^{*}$ мероморфна по $\gamma$ на $\Gamma \backslash P$ и имеет $g+N$ простых нулей в точках $\gamma_{1}, \ldots, \gamma_{g}, R_{1}^{-}, \ldots, R_{N}^{-}$

$\left.2^{*}\right)\left.\operatorname{res} \psi^{*}(\lambda, x, y, t, \tau)\right|_{\lambda=R_{l}^{-}}=-\left.\tau_{l} \frac{\psi^{*}(\lambda, x, y, t, \tau)}{d \lambda}\right|_{\lambda=R_{l}^{+}}, l=1, \ldots, N$;

$\left.3^{*}\right) \psi^{*}$ имеет существенную особенность в точке $P$ и

$$
\psi^{*}(\gamma, x, y, t, \tau)=e^{-k x-k^{2} y-k^{3} t}(1+o(1)) d k \quad \text { при } \gamma \rightarrow P .
$$

Положим

$$
u(x, y, t)=2 \frac{\partial}{\partial x} \xi_{1}(x, y, t, \tau), \quad \tau_{l}=\alpha_{l}+\beta_{l} t, \quad l=1, \ldots, N .
$$

Аналогично по функциям $\psi(\gamma, x, y, t, \tau)$ и $\psi^{*}(\gamma, x, y, t, \tau)$ построим $\psi(\gamma, x, y, t)$ и формы $\psi^{*}(\gamma, x, y, t)$.

Имеет место следующая теорема.

Теорема 8 [4]. Функиия $u(x, y, t)$ вида (34) удовлетворяет уравнению КП с $N$ самосогласованными источниками:

$$
u_{t}=K P[u]+\left.2 \frac{\partial}{\partial x} \sum_{l=1}^{N} \beta_{l} \frac{\psi\left(R_{l}^{-}, x, y, t\right) \psi^{*}(\lambda, x, y, t)}{d \lambda}\right|_{\lambda=R_{l}^{+}},
$$

где $u_{t}=\mathrm{KP}[u]-$ поток Кадомцева-Петвиашвили.

Если существует двулистное накрытие $\pi: \Gamma \rightarrow \mathbb{C} P^{1}$, разветвленное в точке $P, \pi(P)=\infty, \pi(k)=\pi(-k) u \pi\left(R_{l}^{+}\right)=\pi\left(R_{l}^{-}\right), l=1, \ldots, N$, то формула (35) задает решение $u(x, t)$ уравнения КдФ с самосогласованными источниками (в (35) надо заменить $\mathrm{KP}[u]$, на $\mathrm{KDV}[u]$, где $u_{t}=\mathrm{KDV}[u]$ - уравнение $K \partial \Phi)$.

Аналогично можно получить конечнозонные решения всех уравнений из иерархий КдФ и КП с самосогласованными источниками [4]. Доказательство этой теоремы существенно опирается на теорию ядер Коши-Бейкера-Ахиезера, введенных П. Г. Гриневичем и А. Ю. Орловым [47].

ЗАмЕчАниЕ 2. Функция Бейкера-Ахиезера $\psi$ из данной теоремы определена на спектральной кривой с двойными точками $R_{l}$, которая получается из $Г$ попарным склеиванием точек $R_{l}^{+}$и $R_{l}^{-}, l=1, \ldots, N$. Двойная точка $R_{l}$ расклеивается, если и только если $\tau_{l}=0$. Если даже для начального потенциала $u(x, y, t), t=0$, спектральная кривая регулярна, то уравнения с самосогласованными источниками немедленно приводят к появлению двойных точек

\footnotetext{
${ }^{10}$ Сопряженные 1-формы Бейкера-Ахиезера были введены в [46]. Доказательство их существования и единственности аналогично доказательству этих фактов для обычных функций Бейкера-Ахиезера.
} 
на спектральной кривой (их число для почти всех времен равно числу источников $N$ ) и эти сингулярности сохраняются для почти всех моментов времени.

Пример 13. Пусть $\Gamma=\mathbb{C} \cup\{\infty\}=\mathbb{C} P^{1}$ с параметром $k, N=1$ и $R^{ \pm}= \pm \varkappa$. После несложных вычислений получаем формулу

$$
u(x, t, \tau)=-\frac{16 \tau \varkappa^{3}}{\tau e^{-\left(\varkappa x+\varkappa^{3} t\right)}+2 \varkappa e^{\varkappa x+\varkappa^{3} t}},
$$

которая задает (регулярный) солитон при $\tau>0$, нулевое решение при $\tau=0$ и сингулярный солитон при $\tau<0$. Отсюда выводим, что функция $u(x, t)=$ $u(x, t, \alpha+\beta t)$ является решением уравнения КдФ с самосогласованным источником

$$
u_{t}=\frac{1}{4} u_{x x x}-\frac{3}{2} u u_{x}+2 \beta \partial_{x} \psi^{2}(-\varkappa, x, t),
$$

где

$$
\psi(-\varkappa, x, t)=\left(1-\frac{\tau}{\tau+2 \varkappa e^{2\left(\varkappa x+\varkappa^{3} t\right)}}\right) e^{-\varkappa x-\varkappa^{3} t}, \quad \tau=\alpha+\beta t .
$$

Имеют место следующие качественные эффекты, которые были впервые замечены В. К. Мельниковым [43]:

1) стартуя с малого начального значения $c=c(0)$, мы за конечное время достигнем $c=0$ (аннигиляция солитона);

2) при обращении потока (36) при начальных данных $c(0)=0$ мы немедленно получаем солитон при $t>0$ (рождение солитона).

Эти эффекты для быстроубывающих потенциалов являются аналогами исчезновения и рождения двойной точки у спектральных кривых двоякопериодических потенциалов.

\section{5. Некоторые другие примеры интегрируемых задач с сингулярными спектральными кривыми}

Приведем еще пару интересных примеров интегрируемых физических задач с сингулярными спектральными кривыми.

1) Недавно П. Г. Гриневич, А. Е. Миронов и С. П. Новиков выделили класс алгебро-геометрических спектральных данных, по которым с помощью двухточечных функций Бейкера-Ахиезера, введенных в [7], строятся операторы

$$
L=(\partial+A) \bar{\partial}
$$

(магнитный двумерный оператор Паули) [48]. Спектральная кривая Г распадается на две гладкие компоненты $\Gamma^{\prime}$ и $\Gamma^{\prime \prime}$, из которых она получается отождествлением $k+1$ пар точек $Q_{1}^{\prime} \sim Q_{1}^{\prime \prime}, \ldots, Q_{k+1}^{\prime} \sim Q_{k+1}^{\prime \prime}, Q_{i}^{\prime} \in \Gamma^{\prime}, Q_{j}^{\prime \prime} \in \Gamma^{\prime \prime}$, $i, j=1, \ldots, k+1$. При этом существует антиголоморфная инволюция $\sigma: \Gamma \rightarrow \Gamma$, $\sigma^{2}(\gamma)=\gamma, \gamma \in \Gamma$, переставляющая неприводимые компоненты:

$$
\sigma\left(\Gamma^{\prime}\right)=\Gamma^{\prime \prime}, \quad \sigma\left(Q_{k}^{\prime}\right)=Q_{\sigma(k)}^{\prime \prime} .
$$

Отсюда следует, что $\Gamma^{\prime}$ и $\Gamma^{\prime \prime}$ имеют один и тот же род $g$, а $p_{a}(\Gamma)=2 g+k$. Физически интересные примеры операторов возникают уже в случае, когда $\Gamma^{\prime}$ и $\Gamma^{\prime \prime}$ - комплексные проективные кривые. 
С другой вещественной редукцией оператора $L$ :

$$
\widetilde{L}=\left(\partial_{x}+A\right) \partial_{y},
$$

строящейся по таким же сингулярным спектральным кривым, связана интегрируемая двумеризация уравнения Бюргерса [49].

2) В начале 1980-х годов Кричевер применил алгебро-геометрические методы к исследованию решений уравнения Янга-Бакстера на $(4 \times 4)$-матрицы [50]. Построение решений не использует функций Бейкера-Ахиезера, но общая идеология взята из конечнозонного интегрирования и роль спектральной кривой $Г$ играет гладкая эллиптическая кривая. Решения классифицируются по их рангу (он принимает значения $l=1,2)$, и, как показано в [50], все решения ранга 1 калибровочно эквивалентны решению Бакстера, либо получаются из него простыми симметриями. В.И. Драгович рассмотрел вырожденные случаи этой конструкции и показал, что если Г распадается в две рациональные компоненты, пересекающиеся по двум точкам, то получается известное решение Янга [51], а если $Г$ - рациональная кривая с двойной точкой, то мы получаем решение Чередника [52] (в обоих случаях - с точностью до калибровочной эквивалентности).

\section{Список литературы}

[1] А. Е. Миронов, И. А. Тайманов, “Ортогональные криволинейные системы координат, отвечающие сингулярным спектральным кривым”, Функциональные пространства, теория приближений, нелинейный анализ, Сб. статей, Тр. МИАН, 255, Наука, М., 2006, 180-196; англ. пер.: А. E. Mironov, I. A. Taimanov, "Orthogonal curvilinear coordinate systems corresponding to singular spectral curves", Proc. Steklov Inst. Math., 255:1 (2006), 169-184.

[2] А. Е. Миронов, И. А. Тайманов, "О некоторых алгебраических примерах фробениусовых многообразий”, ТMФ, 151:2 (2007), 195-206; англ. пер.: А. Е. Mironov, I. A. Taimanov, "Some algebraic examples of Frobenius manifolds", Theoret. and Math. Phys., 151:2 (2007), 604-613.

[3] P. G. Grinevich, I. A. Taimanov, "Infinitesimal Darboux transformations of the spectral curves of tori in the four-space", Int. Math. Res. Not. IMRN, 2007, rnm005, $22 \mathrm{pp}$.

[4] P. G. Grinevich, I. A. Taimanov, "Spectral conservation laws for periodic nonlinear equations of the Melnikov type", Geometry, topology, and mathematical physics, Amer. Math. Soc. Transl. Ser. 2, 224, Amer. Math. Soc., Providence, RI, 2008, 125-138; arXiv: 0801.4143.

[5] И. М. Кричевер, “Алгебро-геометрические $n$-ортогональные криволинейные системы координат и решения уравнений ассоциативности”, Функи. анализ и его прил., 31:1 (1997), 32-50; англ. пер.: I. M. Krichever, "Algebraic-geometric $n$-orthogonal curvilinear coordinate systems and solutions of the associativity equations", Funct. Anal. Appl., 31:1 (1997), 25-39.

[6] С. П. Новиков, "Периодическая задача для уравнения Кортевега-де Фриза. I", Функи. анализ и его прил., 8:3 (1974), 54-66; англ. пер.: S. P. Novikov, "The periodic problem for the Korteweg-de Vries equation", Funct. Anal. Appl., 8:3 (1974), 236-246.

[7] Б. А. Дубровин, И. М. Кричевер, С. П. Новиков, "Уравнение Шредингера в периодическом поле и римановы поверхности", Докл. АН ССCP, 229:1 (1976), 15-18; 
англ. пер.: В. A. Dubrovin, I. M. Krichever, S. P. Novikov, "The Schrödinger equation in a periodic field and Riemann surfaces", Soviet Math. Dokl., 17 (1976), 947-951.

[8] Б. А. Дубровин, В.Б. Матвеев, С.П. Новиков, "Нелинейные уравнения типа Кортевега-де Фриза, конечнозонные линейные операторы и абелевы многообразия", УМН, 31:1 (1976), 55-136; англ. пер.: В. А. Dubrovin, V. B. Matveev, S.P. Novikov, "Non-linear equations of Korteweg-de Vries type, finite-zone linear operators, and Abelian varieties", Russian Math. Surveys, 31:1 (1976), 59-146.

[9] И. М. Кричевер, "Методы алгебраической геометрии в теории нелинейных уравнений", УМН, 32:6 (1977), 183-208; англ. пер.: I. M. Krichever, "Methods of algebraic geometry in the theory of non-linear equations", Russian Math. Surveys, 32:6 (1977), 185-213.

[10] И. М. Кричевер, С.П. Новиков, "Голоморфные расслоения над алгебраическими кривыми и нелинейные уравнения", УМН, 35:6 (1980), 47-68; англ. пер.: I. M. Krichever, S.P. Novikov, "Holomorphic bundles over algebraic curves and non-linear equations", Russian Math. Surveys, 35:6 (1980), 53-79.

[11] Б. А. Дубровин, “Тэта-функции и нелинейные уравнения”, УМН, 36:2 (1981), 11-80; англ. пер.: В. A. Dubrovin, "Theta functions and non-linear equations", Russian Math. Surveys, 36:2 (1981), 11-92.

[12] И. М. Кричевер, "Спектральная теория двумерных периодических операторов и ее приложения", УМH, 44:2 (1989), 121-184; англ. пер.: I. M. Krichever, "Spectral theory of two-dimensional periodic operators and its applications", Russian Math. Surveys, 44:2 (1989), 145-225.

[13] И.А. Тайманов, “Двумерный оператор Дирака и теория поверхностей”, УМH, 61:1 (2006), 85-164; англ. пер.: I. A. Taimanov, "Two-dimensional Dirac operator and the theory of surfaces", Russian Math. Surveys, 61:1 (2006), 79-159.

[14] И. А. Тайманов, "Представление Вейерштрасса замкнутых поверхностей в $\mathbb{R}^{3}$ ", Функи. анализ и его прил., 32:4 (1998), 49-62; англ. пер.: I. A. Taimanov, "The Weierstrass representation of closed surfaces in $\mathbb{R}^{3 "}$, Funct. Anal. Appl., 32:4 (1998), 258-267.

[15] I. A. Taimanov, "Modified Novikov-Veselov equation and differential geometry of surfaces", Solitons, geometry, and topology: on the crossroad, Amer. Math. Soc. Transl. Ser. 2, 179, Amer. Math. Soc., Providence, RI, 1997, 133-151.

[16] И. М. Кричевер, "Алгебро-геометрическое построение уравнений ЗахароваШабата и их периодических решений", Докл. АН СССР, 227:2 (1976), 291-294; англ. пер.: I. M. Krichever, "Algebraic-geometric construction of the Zakharov-Shabat equations and their periodic solutions", Soviet Math. Dokl., 17 (1976), 39-397.

[17] Ж. Серр, Алгебраические группы и поля классов, Мир, М., 1968, 285 с.; пер. с франц.: J.-P. Serre, Groupes algébriques et corps de classes, Actualités scientifiques et industrielles, 1264, Publications de l'Institut de mathématique de l'Université de Nancago, VII, Hermann, Paris, 1959, 202 pp.

[18] Б. А. Дубровин, И. М. Кричевер, Т. М. Маланюк, В. Г. Маханьков, “Точные решения нестационарного уравнения Шредингера с самосогласованными потенциалами", Физика элементарных частич и атомного ядра, 19:3 (1988), 579-621; англ. пер.: В. A. Dubrovin, T. M. Malanyuk, I. M. Krichever, V. G. Makhankov, "Exact solutions of the time-dependent Schrödinger equation with self-consistent potentials", Soviet J. Particles and Nuclei, 19:3 (1988), 252-269.

[19] И. А. Тайманов, "О двумерных конечнозонных потенциальных операторах Шредингера и Дирака с особыми спектральными кривыми", Сиб. матем. журн., 44:4 (2003), 870-882; англ. пер.: I. A. Taimanov, "On two-dimensional finite-gap potential Schrödinger and Dirac operators with singular spectral curves", Siberian Math. J., 44:4 (2003), 686-694. 
[20] Т.М. Маланюк, "Об одном классе точных решений уравнения КадомцеваПетвиашвили", УМH, 46:3 (1991), 193-194; англ. пер.: Т. M. Malanyuk, "A class of exact solutions of the Kadomtsev-Petviashvili equation", Russian Math. Surveys, 46:3 (1991), 225-227.

[21] Б. А. Дубровин, С.П. Новиков, "Гамильтонов формализм одномерных систем гидродинамического типа и метод усреднения Боголюбова-Уизема", Докл. AH CCCP, 270:4 (1983), 781-785; англ. пер.: В. A. Dubrovin, S. P. Novikov, "Hamiltonian formalism of one-dimensional systems of the hydrodynamic type and the Bogolyubov-Whitham averaging method", Soviet Math. Dokl., 27:3 (1983), 665-669.

[22] С.П. Новиков, "Геометрия консервативных систем гидродинамического типа. Метод усреднения для теоретико-полевых систем", УМН, 40:4 (1985), 79-89; англ. пер.: S.P. Novikov, "The geometry of conservative systems of hydrodynamic type. The method of averaging for field-theoretical systems", Russian Math. Surveys, 40:4 (1985), 85-98.

[23] Б. А. Дубровин, С. П. Новиков, "Гидродинамика слабо деформированных солитонных решеток. Дифференциальная геометрия и гамильтонова теория", УМН, 44:6 (1989), 29-98; англ. пер.: В. A. Dubrovin, S. P. Novikov, "Hydrodynamics of weakly deformed soliton lattices. Differential geometry and Hamiltonian theory", Russian Math. Surveys, 44:6 (1989), 35-124.

[24] V.E. Zakharov, "Description of the $n$-orthogonal curvilinear coordinate systems and Hamiltonian integrable systems of hydrodynamic type. I: Integration of the Lamé equation", Duke Math. J., 94:1 (1998), 103-139.

[25] С. П. Царев, "О скобках Пуассона и одномерных гамильтоновых системах гидродинамического типа", Докл. АН ССCP, 282:3 (1985), 534-537; англ. пер.: S.P. Tsarev, "Poisson brackets and one-dimensional Hamiltonian systems of hydrodynamic type", Soviet Math. Dokl., 31:3 (1985), 488-491.

[26] Б.А. Дубровин, "K дифференциальной геометрии сильно интегрируемых систем гидродинамического типа", Функи. анализ и его прил., 24:4 (1990), 25-30; англ. пер.: В. A. Dubrovin, "Differential geometry of strongly integrable systems of hydrodynamic type", Funct. Anal. Appl., 24:4 (1990), 280-285.

[27] М.В. Павлов, "Интегрируемость егоровских систем гидродинамического типа", TMФ, 150:2 (2007), 263-285; англ. пер.: M. V. Pavlov, "Integrability of the Egorov systems of hydrodynamic type", Theoret. Math. Phys., 150:2 (2007), 225-243.

[28] О. И. Мохов, Е. В. Ферапонтов, “О нелокальных гамильтоновых операторах гидродинамического типа, связанных с метриками постоянной кривизны”, УМН, 45:3 (1990), 191-192; англ. пер.: O. I. Mokhov, E. V. Ferapontov, "Non-local Hamiltonian operators of hydrodynamic type related to metrics of constant curvature", Russian Math. Surveys, 45:3 (1990), 218-219.

[29] G. Darboux, Leçons sur les systèmes orthogonaux et les coordonnées curvilignes, Gauthier-Villars, Paris, 1910, vii+567 pp.

[30] В. Е. Захаров, С. В. Манаков, "О редукциях в системах, интегрируемых методом обратной задачи", Докл. РАН, 360:3 (1998), 324-327; англ. пер.: V. E. Zakharov, S. V. Manakov, "On reductions in systems integrable by the method of the inverse scattering problem", Dokl. Math., 57:3 (1998), 471-474.

[31] Д. А. Бердинский, И. П. Рыбников, "Об ортогональных криволинейных системах координат в пространствах постоянной кривизны”, Сиб. матем. журн. (в печати).

[32] J. Cieśliński, A. Doliwa, P. M. Santini, "The integrable discrete analogues of orthogonal coordinate systems are multi-dimensional circular lattices", Phys. Lett. A, 235:5 (1997), 480-488. 
[33] А. А. Ахметшин, Ю. С. Вольвовский, И. М. Кричевер, “Дискретные аналоги метрик Дарбу-Егорова", Солитоны, геометрия, топология - на перекрестках, Сб. статей. K 60-летию со дня рождения академика Сергея Петровича Новикова, Тр. МИАН, 225, Наука, М., 1999, 21-45; англ. пер.: А. A. Akhmetshin, Yu. S. Vol'vovskij, I. M. Krichever, "Discrete analogs of the Darboux-Egorov metrics", Proc. Steklov Inst. Math., 225 (1999), 16-39.

[34] E. Witten, "On the structure of the topological phase of two-dimensional gravity", Nuclear Phys. B, 340:2-3 (1990), 281-332.

[35] R. Dijkgraaf, E. Verlinde, H. Verlinde, "Topological strings in $d<1$ ", Nuclear Phys. B, 352:1 (1991), 59-86.

[36] B. Dubrovin, "Geometry of 2D topological field theories", Integrable systems and quantum groups (Montecatini Terme, 1993), Lecture Notes in Math., 1620, Springer, Berlin, 1996, 120-348.

[37] Б. А. Дубровин, "Гамильтоновы уравнения в частных производных и фробениусовы многообразия", УМH, 63:6 (2008), 7-18; англ. пер.: В. A. Dubrovin, "Наmiltonian PDEs and Frobenius manifolds", Russian Math. Surveys, 63:6 (2008), 999-1010.

[38] S. Barannikov, M. Kontsevich, "Frobenius manifolds and formality of Lie algebras of polyvector fields", Int. Math. Res. Not., 1998, № 4, 201-215.

[39] V. Shramchenko, "Real doubles' of Hurwitz Frobenius manifolds", Comm. Math. Phys., 256:3 (2005), 635-680.

[40] B. Dubrovin, "Integrable systems in topological field theory", Nuclear Phys. B, 379:3 (1992), 627-689.

[41] В. К. Мельников, "Некоторые новые нелинейные эволюционные уравнения, интегрируемые методом обратной задачи", Матем. сб., 121(163):4(8) (1983), 469-498; англ. пер.: V. K. Mel'nikov, "Some new nonlinear evolution equations integrable by the inverse problem method", Math. USSR-Sb., 49:2 (1984), 461-489.

[42] V.E. Zakharov, E. A. Kuznetsov, "Multiscale expansions in the theory of systems integrable by the inverse scattering transform", Phys. D, 18:1-3 (1986), 455-463.

[43] V.K. Mel'nikov, "Capture and confinement of solitons in nonlinear integrable systems", Comm. Math. Phys., 120:3 (1989), 451-468.

[44] P. G. Grinevich, M. U. Schmidt, "Conformal invariant functionals of immersions of tori into $\mathbb{R}^{3 ",}$ J. Geom. Phys., 26:1-2 (1997), 51-78.

[45] I. A. Taimanov, "Finite gap theory of the Clifford torus", Int. Math. Res. Not., 2005, № $2,103-120$.

[46] И. М. Кричевер, “Модель Пайерлса”, Функи. анализ и его прил., 16:4 (1982), 10-26; англ. пер.: I. M. Krichever, "The Peierls model", Funct. Anal. Appl., 16:4 (1982), 248-263.

[47] P. G. Grinevich, A. Yu. Orlov, "Virasoro action on Riemann surfaces, Grassmannians, $\operatorname{det} \bar{\partial}_{J}$ and Segal-Wilson $\tau$-function", Problems of modern quantum field theory (Alushta, 1989), Res. Rep. Phys., Springer, Berlin, 1989, 86-106.

[48] П. Г. Гриневич, А.Е. Миронов, С.П. Новиков, "О нулевом уровне чисто магнитного двумерного нерелятивистского оператора Паули для частиц со спином 1/2", ТМФ, 164:3 (2010), 333-353; англ. пер.: P. G. Grinevich, A. E. Mironov, S. P. Novikov, "Zero level of a purely magnetic two-dimensional nonrelativistic Pauli operator for spin-1/2 particles", Theoret. and Math. Phys., 164:3 (2010), 1110-1127.

[49] П. Г. Гриневич, А.Е. Миронов, С. П. Новиков, “Двумерный оператор Шрёдингера: эволюционные $(2+1)$-системы и их новые редукции; двумерная иерархия Бюргерса и данные обратной задачи", УМН, 65:3 (2010), 195-196; англ. пер.: P. G. Grinevich, A. E. Mironov, S.P. Novikov, "2D-Schrödinger operator, $(2+1)$ evolution systems and new reductions, 2D-Burgers hierarchy and inverse problem data", Russian Math. Surveys, 65:3 (2010), 580-582. 
[50] И. М. Кричевер, "Уравнения Бакстера и алгебраическая геометрия”, Функи. анализ и его прил., 15:2 (1981), 22-35; англ. пер.: I. M. Krichever, "Baxter's equations and algebraic geometry", Funct. Anal. Appl., 15:2 (1981), 92-103.

[51] В.И.Драгович, "Решения уравнения Янга с рациональными спектральными кривыми", Алгебра и анализ, 4:5 (1992), 104-116; англ. пер.: V. I. Dragovich, "Solutions of the Yang equation with rational spectral curves", St. Petersburg Math. J., 4:5 (1993), 921-931.

[52] В.И. Драгович, "Решения уравнения Янга с рациональными неприводимыми спектральными кривыми", Изв. РАН. Сер. матем., 57:1 (1993), 59-75; англ. пер.: V.I. Dragovich, "Solutions of the Yang equation with rational irreducible spectral curves", Russian Acad. Sci. Izv. Math., 42:1 (1994), 51-65.

И. А. Тайманов (I. A. Taimanov)

Институт математики им. С. Л. Соболева СО РАН

E-mail: taimanov@math.nsc.ru
Поступила в редакцию 01.12 .2010 